\title{
A numerical investigation into the aerodynamic characteristics and aeroelastic
} stability of a footbridge.

\author{
I J Taylor ${ }^{\mathrm{a},{ }^{*}}$, M Vezza ${ }^{\mathrm{b}}$ \\ a Dept of Mechanical Engineering, University of Strathclyde, Glasgow, G1 1XJ. \\ ${ }^{\mathrm{b}}$ Dept of Aerospace Engineering, University of Glasgow, Glasgow, G12 8QQ.
}

\begin{abstract}
:
The results of a numerical investigation into the aerodynamic characteristics and aeroelastic stability of a proposed footbridge across a highway in the north of England are presented. The longer than usual span, along with the unusual nature of the pedestrian barriers, indicated that the deck configuration was likely to be beyond the reliable limits of the British design code BD 49/01. The calculations were performed using the discrete vortex method, DIVEX, developed at the Universities of Glasgow and Strathclyde. DIVEX has been successfully validated on a wide range of problems, including the aeroelastic response of bridge deck sections. In particular, the investigation focussed on the effects of non-standard pedestrian barriers on the structural integrity of the bridge. The proposed deck configuration incorporated a barrier comprised of angled flat plates, and the bridge was found to be unstable at low wind speeds with the plates having a strong turning effect on the flow at the leading edge of the deck. These effects are highlighted in both a static and dynamic analysis of the bridge deck, along with modifications to the design that aim to improve the aeroelastic stability of the deck. Proper orthogonal decomposition (POD) was also used to investigate the unsteady pressure field on the upper surface of the static bridge deck. The results of the flutter investigation and the POD analysis highlight the strong influence of the pedestrian barriers on the overall aerodynamic characteristics and aeroelastic stability of the bridge.
\end{abstract}

Keywords : Flutter; Bridge; Vortex Method; CFD; Pedestrian Barrier; Proper Orthogonal Decomposition.

\section{INTRODUCTION}

In the early design stages for bridges, engineers will seek to assess aerodynamic loading from the available design codes, for example British Standard BD 49/01. However, these codes use analysis methods and empirical correlations that are largely based on experience from previous designs. Hence, many of the new and innovative designs lie beyond the scope of current design codes, with assessment of the aeroelastic stability of the bridge relying on alternative means.

Engineers are now increasingly using numerical methods for aerodynamic analysis particularly in the initial stages of the design, allowing designers to assess a range of

\footnotetext{
* Corresponding author: Department of Mechanical Engineering, James Weir Building, Montrose Street, University of Strathclyde, Glasgow, G1 1XJ, UK.

Tel.: +44 (0)141 548 3753; Fax: +44 (0)141 5525105 Email address : ian.taylor@ strath.ac.uk
} 
potential design options, to perform feasibility studies on novel configurations, or to provide useful aerodynamic and structural information on designs that lie beyond the scope of design codes. This is particularly useful on configurations for which there is no previous design knowledge or experience.

A particular numerical technique that in recent years has been proven to be extremely well suited to assessing unsteady aerodynamic effects and structural integrity is the discrete vortex method. Unlike traditional grid based CFD techniques, such as finite volume, finite difference or finite element, the vortex method approach has demonstrated sufficient accuracy over a range of applications (Sweeney and Meskell, 2003; Akbari and Price, 2003 and 2005 ), as well as being computationally efficient so that results can be obtained relatively quickly. These factors combined having led to a number of researchers being able to apply the vortex method to real designs, with the numerical procedure used very successfully as a design tool within bridge deck design procedures (Larsen and Walther, 1997; MacKenzie et al., 2002; Taylor et al., 2002b; Vejrum et al., 2000).

Discrete vortex methods are based on the discretisation of the vorticity field rather than the velocity field, into a series of vortex particles, each of which is of finite core size and carrying a certain amount of circulation. The particles are tracked in time throughout the flow field that they collectively induce. As a result of this approach, the model does not require a calculation mesh and provides a very different method of analysis to more traditional grid based computational fluid dynamics methods. The Lagrangian nature of the method significantly reduces some of the problems that are associated with grid based methods. These primarily include numerical diffusion and difficulties in achieving resolution of small scale vortical structures in the flow. Comprehensive reviews of the discrete vortex method are given in Leonard (1980 and 1985), and Sarpkaya (1989).

The two dimensional discrete vortex method, DIVEX, developed at the Universities of Glasgow and Strathclyde has been used to analyse a wide range of bluff body flow fields. These include both static and oscillating sharp edged bodies (Taylor and Vezza, 1999a, 1999b). More recently, investigations into static and oscillating circular cylinders have been undertaken as a preliminary study in the phenomenon of rain-windinduced oscillation (Robertson and Taylor, 2006). DIVEX has also been extensively validated for a range of bridge deck analyses, ranging from predictions of static aerodynamics loads, flutter analysis, and the study of flow control devices (Taylor and Vezza, 2001 and 2002a). A well established tool for bridge sectional aerodynamic studies, DIVEX has been used during a number of recent design projects, providing information ranging from flutter stability and static wind loading on deck sections to providing indications of the performance of wind shielding (Taylor et al., 2002b, 2005).

The results presented herein are based on a design study, commissioned by Halcrow Group Ltd., performed at the Universities of Glasgow and Strathclyde, on a proposed footbridge in the North of England. The initial study focused on assessing the structural integrity of the bridge with respect to flutter and was originally undertaken as the 
proposed crossing was for a span longer than previously experienced for a bridge of this type. Also, the study was commissioned as the pedestrian barriers along the edges of the deck were of a unique design, considered to be outside of the scope of the British design code BD 49/01. The design of the barriers comprised a series of "flat plates" angled down towards the upper surface of the deck.

This paper presents the results of the numerical analysis, investigating the aerodynamic characteristics of the basic deck section as well as three barrier configurations, to demonstrate the strong detrimental effect that these barriers have on the flutter instability. The unsteady pressure field on the upper surface of the deck is analysed using proper orthogonal decomposition (POD), which has increasingly been utilised in wind engineering to describe fluctuating surface pressures around structures (Amandolèse and Crémona, 2005; Chen and Letchford, 2005; Chen and Kareem, 2005; Matsumoto et al. 2006), with a review of the technique provided by Tamura et al., 1999. POD can be used to analyse the temporally and spatially varying data, and provides a means of identifying the various flow phenomena within the fluctuating pressure field and to ascertain the dominant features of the flow field. The POD analysis of the upper deck surface pressures, combined with the predicted aerodynamic characteristics of each deck configuration, illustrate the dominant effect of the pedestrian barriers. The numerical analysis of the mean aerodynamic loads and aeroelastic stability of the proposed bridge deck highlights the usefulness of DIVEX as part of a design process, and more generally demonstrates how numerical procedures can be used to provide information to assist engineers in the assessment of the structural integrity of new and unique designs.

\section{PROPOSED BRIDGE DESIGN}

The proposed bridge is a new pedestrian crossing across a widened highway in the north of England. The design of the bridge is based on a previous design, to build upon and utilise previous knowledge and experience, but also to provide an element of "corporate identity" for the bridge. Two main uncertainties in the proposed design instigated the numerical investigation into the aerodynamic and aeroelastic characteristics of the bridge. Firstly, as the bridge will be crossing a widened highway, it has a span significantly longer than previous similar designs. Hence, the potentially lower structural stiffness of this longer span raised a few concerns over its structural integrity, necessitating the requirement to assess the aeroelastic stability of the proposed bridge with respect to both flutter and vortex induced vibration. Secondly, the existing bridges of this type were not only pedestrian crossings but also part of a bridle path where there would be a significant number of crossings by riders on horseback. For this reason, the barriers along the edges of the deck comprised a series of angled flat plates, designed specifically to block the horse's view of vehicular movement underneath the bridge, thus preventing the horse being startled whilst crossing the bridge. Although the new footbridge is not part of a bridle path, it was decided that the angled flat plates be retained as part of the initial design, to maintain consistency with previous designs. 


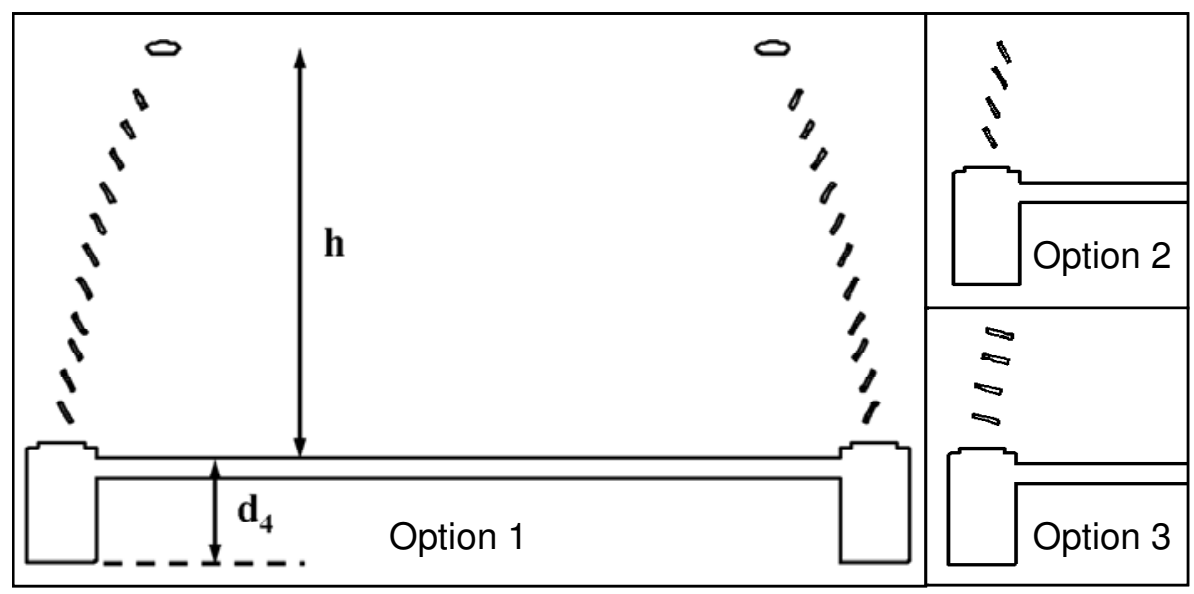

Figure 1. Schematic diagram illustrating basic deck section and three barrier configurations

Table 1. Summary of the design modifications analysed using DIVEX

\begin{tabular}{ll}
\hline Deck Option & Description \\
\hline Basic Deck Section & $\begin{array}{l}\text { No pedestrian barriers - Deck section comprised of } 2 \\
\text { rectangular box section tubes, with concrete slab footway. }\end{array}$ \\
Parapet Option 1 & $\begin{array}{l}\text { Steeply angled flat plates on each side of deck up to the height } \\
\text { of the pedestrian hand rail. ( Initial proposed design ) }\end{array}$ \\
Parapet Option 2 & 4 steep angled flat plates on each side of deck. \\
Parapet Option 3 & 4 shallow angled flat plates on each side of deck. \\
\hline
\end{tabular}

However, the design of these "angled vanes" meant that the configuration of the bridge deck was outside the scope of the design code BD 49/01, providing further justification for performing a numerical analysis to assess the aerodynamic characteristics and susceptibility of the structure to aeroelastic phenomena.

In the numerical analysis, three different barrier configurations were investigated, as illustrated in Fig. 1, along with the basic deck section with no pedestrian barriers to provide a datum case for comparison. A summary of the four bridge deck options is given in Table 1, with some structural properties of the bridge indicated in Table 2. The initial proposed barrier design (Option 1) comprised the pedestrian barrier of angled plates extending up to the height of the pedestrian hand rail. Two modifications of this design were considered when it became clear that the barriers were having a much stronger than anticipated effect on the aerodynamic characteristics of the structure. These alternative design options were chosen in an attempt to mitigate the strong effect of the angled flat plates, by modifying both the extent of the barriers vertically above the main deck, and reducing the angle of incidence of the barriers with respect to the oncoming flow. 
Table 2. Structural properties of proposed bridge design.

\begin{tabular}{ll}
\hline Structural Properties & Proposed Footbridge \\
\hline Bridge Deck Width, $B$ & $3.8 \mathrm{~m}$ \\
Mass per unit span, $m$ & $1427.6 \mathrm{~kg} / \mathrm{m}$ \\
$\begin{array}{l}\text { Moment of inertia per unit span, } \mathrm{I}_{\alpha} \\
\quad \text { (2nd moment of mass) }\end{array}$ & $1172 \mathrm{kgm}^{2} / \mathrm{m}$ \\
$\begin{array}{l}\text { 1st bending (vertical DOF) frequency, } f_{h} \\
\text { 1st torsional frequency, } f_{\alpha}\end{array}$ & $1.42 \mathrm{~Hz}$ \\
Critical damping ratio, $\zeta_{h}, \zeta_{\alpha}$ & $3.06 \mathrm{~Hz}$ \\
$\quad$ (vertical and torsional DOF) & $0.5 \%$ \\
Density of Air, $\rho$ & $1.225 \mathrm{~kg} / \mathrm{m}^{3}$ \\
\hline
\end{tabular}

Also included in Fig. 1 are relevant dimensions of the barriers and deck, which are used to ascertain the applicability of BD 49/01. Initial concerns over the proposed footbridge were due to the longer than usual span for this bridge design, however, despite this the bridge still lies within the scope of the design rules in BD 49/01. However, when the barriers formed by the angled flat plates are considered, then it is much less clear as to whether the proposed design lies within the scope of BD 49/01. The design guidelines generally consider barriers in the form of bluff objects placed normal to the oncoming flow, and do not incorporate any guidance on barriers of this nature. The design rules place geometric constraints on the applicability of the design code with respect to barriers, relating the barrier dimensions to their solidity (Eq. (1)). The design rules indicate that the solidity ratio of the barrier, $\phi$, should be less than 0.5 and that the product of the barrier height, $h$, (Fig. 1) and $\phi$ should be less than $35 \%$ of the depth of the bridge deck, $d_{4}$ (Section 2.3 - BD 49/01)

$$
\text { Geometric Constraints }\left\{\begin{array}{c}
\phi<0.5 \\
h \phi<0.35 d_{4}
\end{array}\right. \text {. }
$$

The nature of the barriers on the bridge deck, and their orientation at an angle of incidence to the oncoming flow, mean that it is difficult to select an appropriate value of the solidity ratio, $\phi$. To satisfy the geometric constraint, a solidity ratio of less than 0.09 is required to ensure that the product $h \phi$ is less than $35 \%$ of the deck depth, $d_{4}$.

\section{NUMERICAL MODELLING OF FLOW FIELD}

\subsection{Discrete Vortex Method}

The numerical technique used in the analysis was the discrete vortex method, DIVEX, developed at the Universities of Glasgow and Strathclyde. The model is a two- 
dimensional unsteady aerodynamic solver for incompressible and viscous flow fields. In this approach, the vorticity field is discretised into a series of vortex particles, rather than the more traditional approach utilised in grid based CFD where the velocity field is discretised. The detailed numerical implementation of the model is given in Lin, 1997 and Taylor and Vezza, 1999a. DIVEX can be used purely as an aerodynamic tool or in full aeroelastic mode where the response of structures to the unsteady flow can be assessed. In the former case, both static and moving bodies can be modelled as demonstrated in previous analyses, ranging from square and rectangular cylinders to suspension bridge deck sections and flow control devices (Taylor and Vezza, 1999a, 2001, 2002a).

\subsection{Mathematical formulation.}

Two dimensional incompressible flow is governed by the following continuity and full viscous Navier-Stokes equations, in vorticity and stream function form : Continuity equation :

$$
\nabla^{2} \Psi=-\omega .
$$

Vorticity transport equation :

$$
\frac{\partial \boldsymbol{\omega}}{\partial t}+(\mathbf{U} . \nabla) \boldsymbol{\omega}=v \nabla^{2} \boldsymbol{\omega}
$$

The vorticity is defined as the curl of the velocity, $\boldsymbol{\omega}=\nabla \times \mathbf{U}$ with $\boldsymbol{\omega}=\omega \mathbf{k}$, and the vector potential, $\Psi$, is defined such that $\mathbf{U}=\nabla \times \boldsymbol{\Psi}, \quad \boldsymbol{\Psi}=\Psi \mathbf{k}, \quad \nabla . \boldsymbol{\Psi}=0$.

The vorticity transport equation, Eq. (3), defines the motion of vorticity in the flow due to convection and diffusion. As the pressure field is not explicitly defined in Eq. (3), the time variation of vorticity at a point in the flow is therefore dependent on the surrounding flow velocity and vorticity fields.

By using Green's Theorem, the velocity field can be calculated using the Biot-Savart law, which expresses the velocity in terms of the vorticity field. For a point $p$ outside the solid region, the velocity is given by :

$$
\begin{gathered}
\mathbf{U}_{p}=\mathbf{U}_{\infty}+\frac{1}{2 \pi} \int_{F_{b}} \omega \frac{\mathbf{k} \times\left(\mathbf{r}_{p}-\mathbf{r}\right)}{\left\|\mathbf{r}_{p}-\mathbf{r}\right\|^{2}} d F_{b}+\frac{1}{2 \pi} \int_{F_{w}} \omega \frac{\mathbf{k} \times\left(\mathbf{r}_{p}-\mathbf{r}\right)}{\left\|\mathbf{r}_{p}-\mathbf{r}\right\|^{2}} d F_{w} \\
+\frac{1}{2 \pi} \int_{B_{i}} 2 \Omega_{i} \frac{\mathbf{k} \times\left(\mathbf{r}_{p}-\mathbf{r}\right)}{\left\|\mathbf{r}_{p}-\mathbf{r}\right\|^{2}} d B_{i} .
\end{gathered}
$$

Eq. (4) details the four contributions to the velocity at a point $p$ in the flow, from the freestream, the vorticity contained within a small region or "control zone" close to the body surface, the vorticity in the remaining flow field or "wake", and the vorticity in the solid region due to the rotational motion of the body.

The pressure distribution on the body surface can be evaluated by integrating the pressure gradient along the body contour which at node $j$ on the body surface, is given by Eq. (5) (Lin, 1997). 


$$
\frac{1}{\rho} \frac{\partial P}{\partial s}=-\mathbf{s} \cdot \frac{D \mathbf{U}_{c}}{D t}-\mathbf{n} .\left(\mathbf{r}-\mathbf{r}_{c}\right) \frac{D \Omega}{D t}+\mathbf{s} .\left(\mathbf{r}-\mathbf{r}_{c}\right) \Omega^{2}+v \frac{\partial \omega}{d n} .
$$

The first three terms on the RHS in Eq. (5) are due to the body motion and represent the surface tangential components of the body reference point acceleration, the rotational acceleration and the centripetal acceleration. The final term is the negative rate of vorticity creation at the body surface and is calculated from the vorticity distribution created in the control zone between time $t-\Delta t$ and $t$. The resulting pressure distribution is integrated around the body surface to calculate the aerodynamic forces on the body.

\section{PROPER ORTHOGONAL DECOMPOSITION}

The technique of proper orthogonal decomposition has been increasingly used in wind engineering, particularly to investigate unsteady pressure data. In the complex flow fields typical of those experienced in bluff body aerodynamics and flows around buildings, the unsteady pressure records are influenced by various flow phenomena, such as vortex shedding, regions of unsteady separated flow with fluctuating separation points and turbulence effects. POD is a method used to derive the most efficient coordinate system for observing individual flow phenomena, and thus identifies the deterministic or systematic structures contained within the random fluctuating pressure field, and to assist in understanding the nature of each of these flow phenomena. Thus, POD can provide a means of ascertaining and clarifying how different flow phenomena are affecting the overall aerodynamic characteristics of the flow field. A review of the technique for wind engineering applications is given by Tamura et al. (1999).

In the current analysis, the unsteady pressure field on the deck surface is investigated, though the technique can be applied to other fluctuating parameters, such as the velocity field (Chen and Letchford, 2005). Tamura et al. (1999) demonstrates that if the fluctuating pressure, with a zero mean, at a point on the body surface is represented by $p(x, y, t)$, then a deterministic function, $\Phi(x, y)$, can be derived, such that the projection of $p(x, y, t)$ onto the deterministic coordinate function is maximised. Thus, the coordinate function, $\Phi(x, y)$ is found so that it best correlates with all the elements of the unsteady pressure field. For an unsteady pressure field with $N$ "measurement" locations, this leads to an eigenvalue problem, in matrix form :

$$
\mathbf{R}_{P} \boldsymbol{\Phi}=\lambda \boldsymbol{\Phi}
$$

where $\mathbf{R}_{p}$ is a covariance matrix $(N \times N)$ of the fluctuating pressures, with $\Phi$ and $\lambda$ respectively denoting the eigenvectors and eigenvalues of the covariance matrix, $\mathbf{R}_{p}$. As $\mathbf{R}_{p}$ is an $N \times N$ matrix, there are in principle $N$ eigenvectors, $\Phi_{n}(n=1,2, \ldots, n)$, so $N$ deterministic coordinate functions can be found. By utilising the orthogonality of the eigenvectors, the unsteady pressure field can now be expressed as :

$$
p(x, y, t)=\sum_{n=1}^{N} a_{n}(t) \boldsymbol{\Phi}_{n}(x, y),
$$


where $a_{n}(t)$ is the $n$th principal coordinate. It can also easily be shown that, for normalised $\Phi_{n}$

$$
\overline{a_{n}^{2}(t)}=\lambda_{n} \text {. }
$$

The deterministic functions, $\Phi_{n}(x, y)$, found from the eigenvector analysis are also termed the modes of the POD analysis. A measure of the significance of each mode with respect to the overall unsteady flow field, can be determined by considering the ratio of each eigenvalue to the sum of eigenvalues. The proportion of the $n$th principal coordinate or POD mode is defined as

$$
c_{n}=\frac{\lambda_{n}}{\sum_{n=1}^{N} \lambda_{n}},
$$

with the cumulative proportion up to the $N$ th mode defined as

$$
C_{N}=\sum_{n=1}^{N} c_{m} .
$$

As the deterministic function is the coordinate function that best correlates with all the elements of the unsteady pressure field, the lower modes are effectively representing the data in a coordinate system based upon flow phenomena that are most predominant in the overall flow field. Hence, from the lower POD modes, the underlying systematic structure of the flow are their relative importance can be interpreted. Also, from signal analysis of $a_{n}(t)$, the frequencies of the flow structures dominant in each mode can be determined. For example, Matsumoto et al. (2006) illustrates for a flow field dominated by von Karman vortex shedding, the first two eigenmodes have the same frequency, matching the Strouhal frequency of the vortex shedding, and account for over $95 \%$ of the energy within the flow.

\section{STATIC ANALYSIS}

\subsection{Numerical analysis}

Numerical simulations were performed for the each of the bridge deck options, at a range of angles of incidence, between $+5^{\circ}$ and $-5^{\circ}$, from which the static aerodynamic loading could be determined. The mean lift, drag and moment coefficients are respectively defined as:

$$
C_{L}=\frac{L}{\frac{1}{2} \rho U_{\infty}^{2} B} \quad, \quad C_{D}=\frac{D}{\frac{1}{2} \rho U_{\infty}^{2} B} \quad, \quad C_{M}=\frac{M}{\frac{1}{2} \rho U_{\infty}^{2} B^{2}},
$$

where $U_{\infty}$ is the onset wind velocity, $\rho$ is the density of air, $B$ is the deck width, and $L$, $D$ and $M$ are the lift, drag and moment forces. Positive lift is in the upwards direction and positive moment (and angle) is in the clockwise direction (positive moment tends to twist leading edge of the deck upwards) (Fig. 2).

Root mean square (RMS) quantities are also calculated to give a measure of the fluctuation of various parameters about the mean value throughout the analysis. In each 
case, the RMS fluctuation is calculated using Eq. (12), where $C$ is the parameter for which the RMS value is being determined (e.g. pressure coefficient, velocity, aerodynamic force etc. ), and $\mathrm{N}$ is the number of samples (timesteps) from the time history that are used to calculate the mean and RMS quantities.

$$
C_{R M S}=\sqrt{\sum_{i=1}^{N}\left(C_{i}-\bar{C}\right)^{2} / N} .
$$

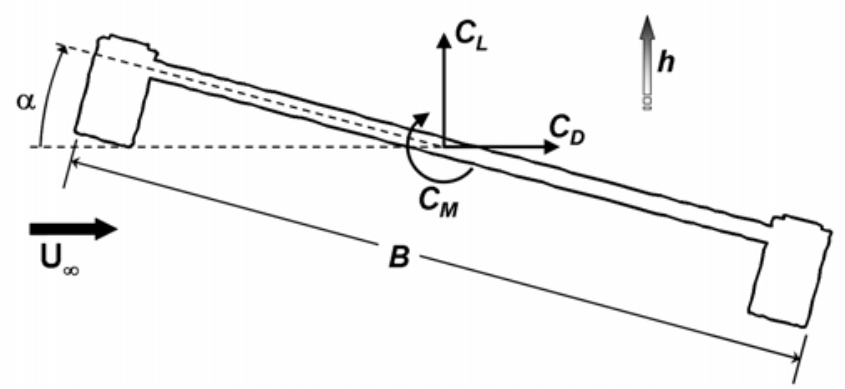

Figure 2. Frame of reference and indication of positive directions for analysis.

\subsection{Aerodynamic Force Coefficients}

Unsteady time histories of the aerodynamic force coefficients are obtained from the DIVEX calculations on the static bridge deck. The calculations are for an impulsively started flow and hence the initial portion of the time history, where the flow field is developing, is omitted from the calculation of the mean aerodynamic force coefficients. The mean aerodynamic loads for the basic deck section and each of the three barrier options at $0^{\circ}$ incidence, are shown in Table 3, along with the slope (per radian) of the lift and moment coefficients at $0^{\circ}$. The variations of lift and moment coefficients with incidence are illustrated in Fig. 3.

Table 3. Mean Aerodynamic Loads and Gradient (per radian) on each Bridge Deck Option at $0^{\circ}$ Incidence.

\begin{tabular}{lccccc}
\hline Configuration & Lift Coef. & Drag Coef. & $\begin{array}{c}\text { Moment } \\
\text { Coef. }\end{array}$ & $\begin{array}{c}\text { Lift slope } \\
\left(\mathrm{rad}^{-1}\right) \\
d C_{L} /\left.d \alpha\right|_{\alpha=0}\end{array}$ & $\begin{array}{c}\text { Moment slope } \\
\left(\mathrm{rad}^{-1}\right)\end{array}$ \\
\hline $\begin{array}{c}\text { Basic deck section } \\
\begin{array}{c}\text { Option } 1 \text { : Full height flat } \\
\text { plates. }\end{array}\end{array}$ & 0.425 & 0.263 & 0.0103 & 11.70 & 1.083 \\
$\begin{array}{c}\text { Option 2 : 4 steep flat } \\
\text { plates. }\end{array}$ & -1.183 & 0.717 & 0.175 & 8.41 & 0.057 \\
$\begin{array}{c}\text { Option 3 : 4 shallow flat } \\
\text { plates. }\end{array}$ & -0.433 & 0.409 & 0.0481 & 10.32 & 0.198 \\
\hline
\end{tabular}




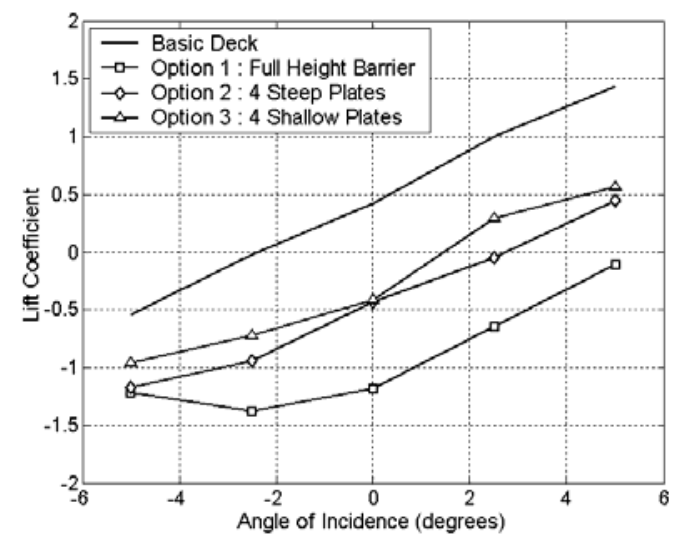

a) Lift Coefficient

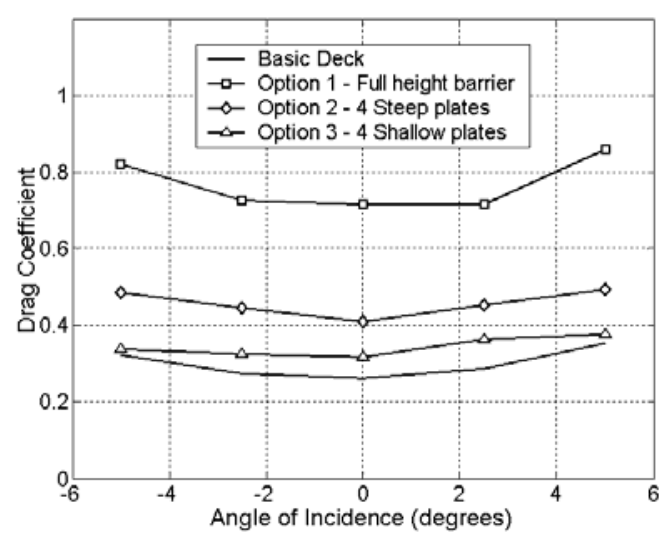

b) Drag Coefficient

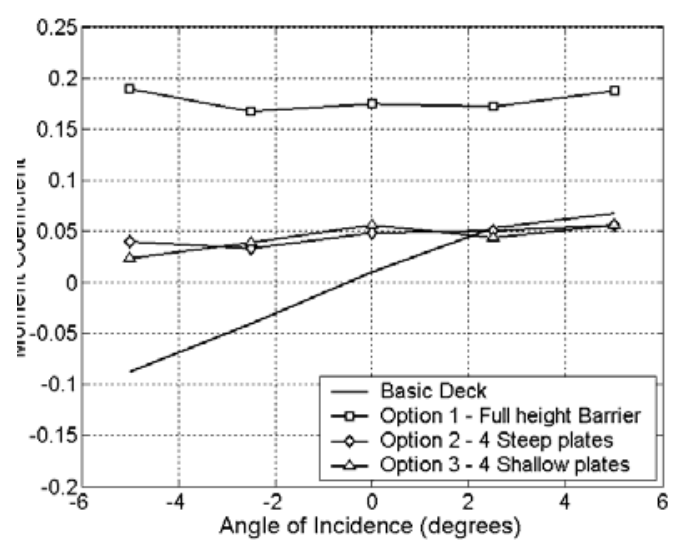

c) Moment Coefficient

Figure 3. Aerodynamic force coefficients for basic deck and barrier options.

The presence of the angled plates has a significant effect on the static loads, noticeably reducing the mean lift coefficient and increasing moment coefficient at $0^{\circ}$. In effect, the flat plate barriers act as guide vanes, redirecting the oncoming flow downwards towards the top surface of the deck, thus preventing the usual development of negative pressure around the leading edge. On the basic deck, the separated flow region produces high suction pressures on the upper deck surface giving rise to the generally positive lift coefficient for the basic deck section. This suction is greatly reduced when the barriers are included due to the flow being directed along the upper surface of the deck, and so there is a reduction in the lift coefficient for all three barrier configurations, with the effect being most pronounced in Option 1, where the barriers extend to full height. An schematic illustration of the effect of the barriers on the flow compared to the basic deck section is shown in Fig. 4.

At angles of incidence away from $0^{\circ}$, the dominant effect of the barriers is clear, with the turning effect of the flat plate barriers ensuring that the flow along the upper surface of the deck remains relatively unchanged, and the variation in upper surface pressures with angle of incidence is significantly reduced compared to the basic deck. This modification of the flow field at the windward and leeward sides of the deck has a significant influence on the aerodynamic loads, particularly as the angle of incidence, $\alpha$, 
is varied. Hence, the proportionate increase in moment with incidence does not occur when the barriers are included, and thus the moment slope is greatly reduced. It should be noted that a low value of moment slope is an indication of potential susceptibility to single degree of freedom torsional instability. Another contributory factor to the change in moment coefficient is due to the plates at the windward side of the deck experiencing an upwards reaction force as the flow is directed downwards toward the upper deck surface. Similarly, the leeward plates experience a downward force as they direct the flow upwards. The combination of these two effects results in a significant increase in moment about the deck centre, as clearly illustrated in Fig. 3c.

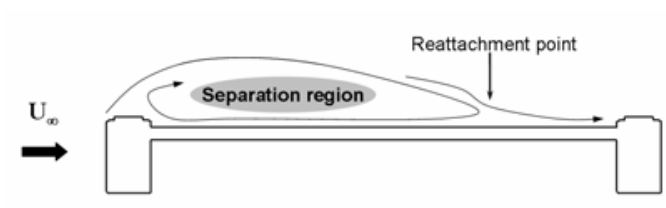

a) Basic Deck Section

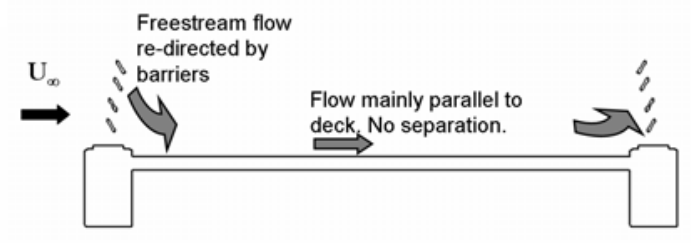

b) Deck with barriers

Figure 4. Schematic diagram to compare of typical flow field for basic deck section, and deck with barriers included.

In general, the drag coefficient follows the expected trends, increasing due to the inclusion of barriers and with angles of incidence away from $0^{\circ}$. Furthermore, the largest increase in drag is for Option 1, where the barriers are full height.

\subsection{Mean and RMS velocity Fluctuations}

The mean velocity field for each deck option at $0^{\circ}$ incidence is illustrated using velocity vectors and contour plots of mean velocity in Figs. 5-6, contours of RMS velocity fluctuations are illustrated in Fig. 7. In each case, the mean velocity is normalised with respect to the freestream flow, which approaches from the left, and all dimensions are normalised with respect to the deck width, $B$.

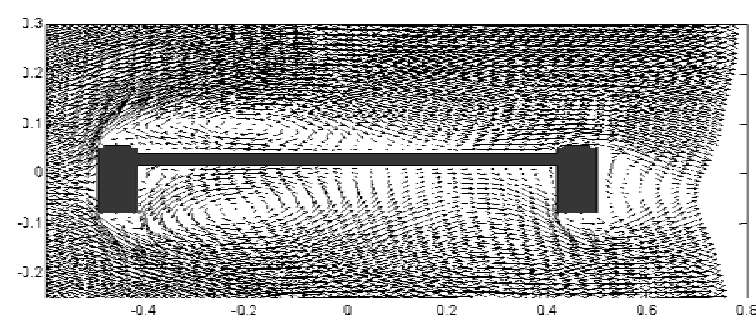

Basic Deck

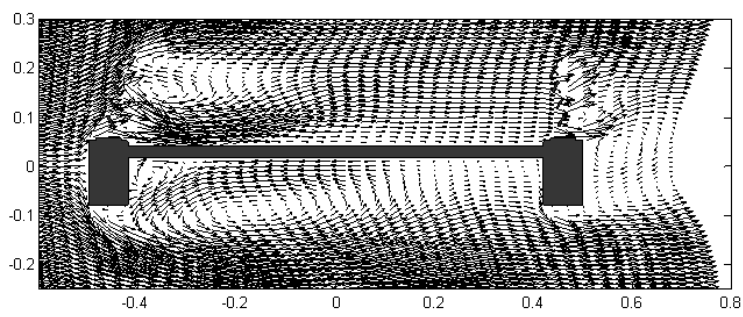

Option 2
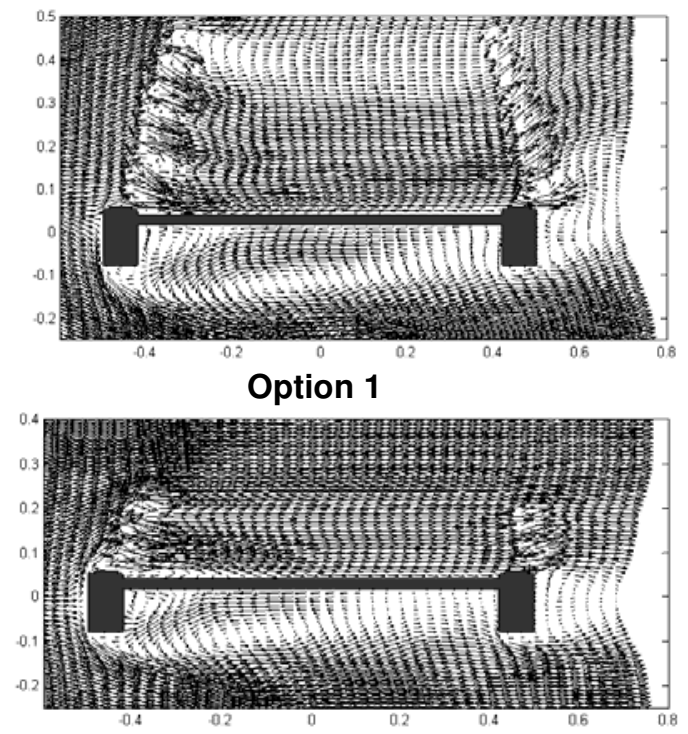

Option 3

Figure 5. Mean velocity vectors for each deck option. 


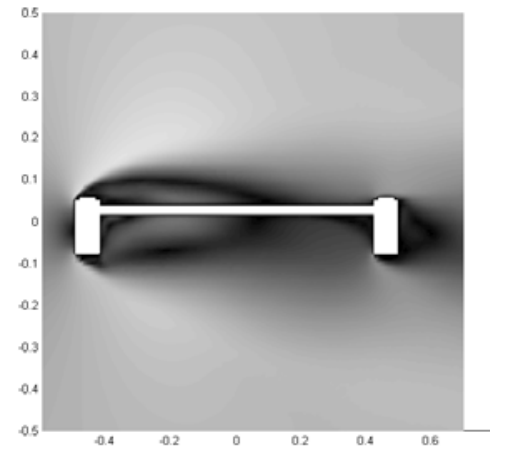

Basic Deck

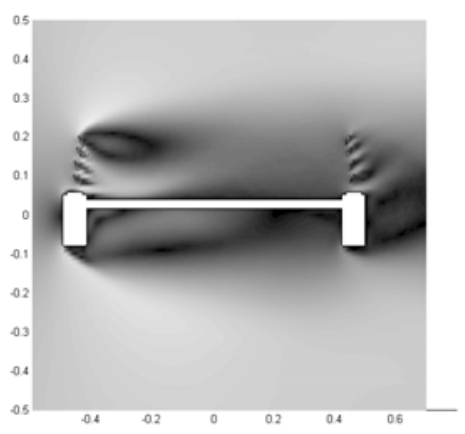

Option 2

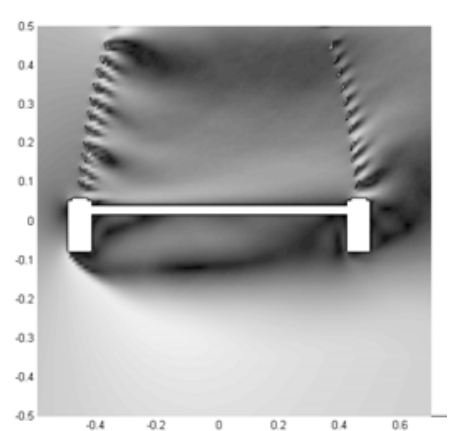

Option 1

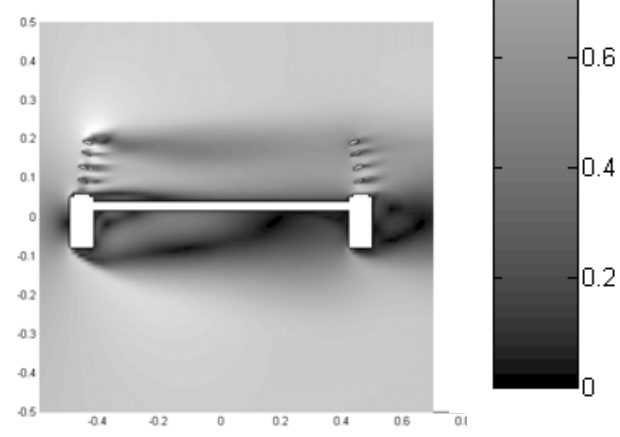

Option 3

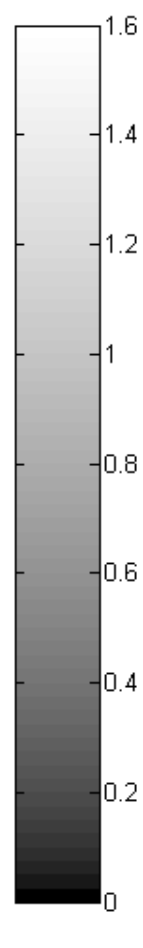

Figure 6. Contours of mean velocity for each deck option.

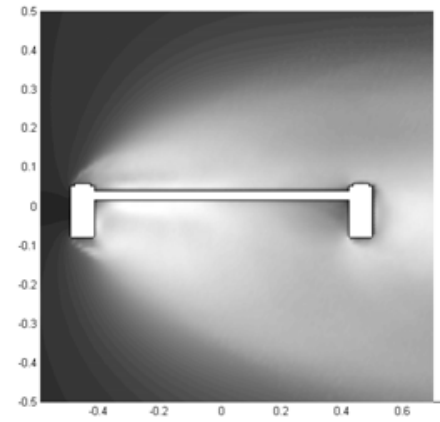

Basic Deck

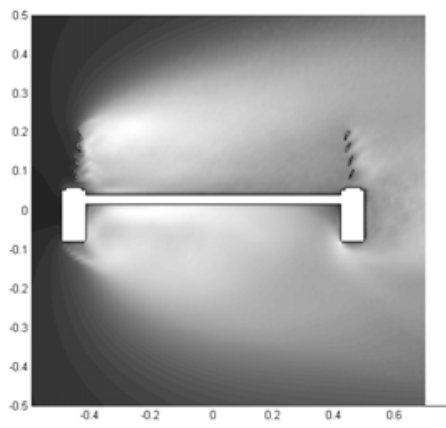

Option 2

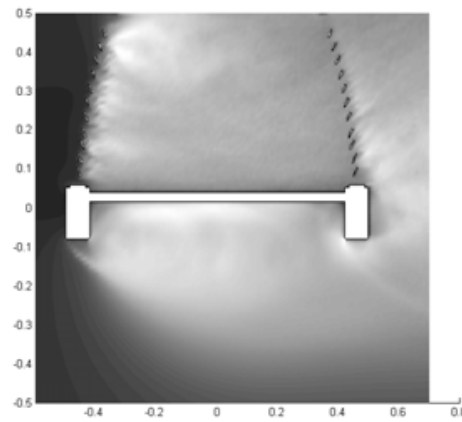

Option 1

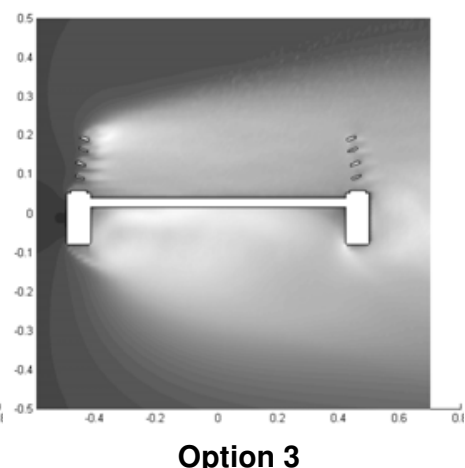

Option 3

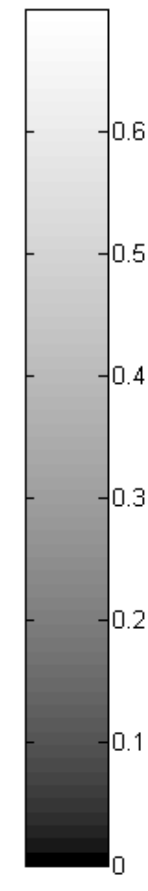

Figure 7. Contours of RMS fluctuating velocity for each deck option. 
The time-averaged flow field around the basic deck section follows the expected pattern, with the flow stagnating on the front face of the deck, and a separated region developing over the upper deck surface, reattaching at $x / B$ of about $0.1-0.2(60-70 \%$ deck width) ${ }^{1}$. There are also large velocity fluctuations in the region of separated flow.

When the pedestrian barriers were included, the strong influence of the flat plates on the flow over the upper surface of the deck is clear in all three barrier configurations. Notably, the velocity vector plot clearly demonstrates the effect of the barrier on the windward side, turning the flow strongly down onto the deck surface. Consequently, the region of separated flow over the upper surface is not present, as the flow has been directed along the upper surface of the deck. Similarly, the flat plates at the leeward edge of the deck deflect the flow strongly upwards. The RMS fluctuations along the upper surface in each case are greatly reduced, further indicating that flow separation has been suppressed. A particular feature of Option 2 is that the steep angled plates seem to generate much greater acceleration of the flow down onto the upper surface of the deck, an effect which is mitigated to a large extent by the reduction in the angle of the flat plates in deck option 3.

\subsection{Mean and RMS Fluctuating Pressure Coefficients}

The mean pressure coefficients and RMS fluctuating pressures for each deck option are shown in Figs. 8-10. In each case, only the data along the upper surface of the deck is illustrated, to enable the effect of the barriers on the pressure distribution to be assessed. The pressure distributions are plotted against the percentage non-dimensional horizontal distance along the deck, normalised with respect to the deck width, $B$, with the deck illustrated along with each pressure plot for reference.

For the basic deck section at $0^{\circ}$ incidence, the separated region of flow is clearly characterised by a region of large suction pressures up to around $x / B=0.1-0.2$, consistent with the results illustrated in the contours of mean velocity. The separated region is also clear in the RMS pressure coefficient, with large fluctuations over most of the upper surface of the deck. In each of the three barrier configurations, the large suction pressures are significantly reduced, and the mean pressure tends to show much less variation over the whole of the upper surface, indicative of the fact that the separation region has been suppressed by the barriers. Also, the RMS pressure fluctuations are significantly reduced by the inclusion of the barriers, again illustrating the strong effect the barriers are having on the flow over the upper surface of the deck. Barrier Option 2 still has a large suction pressure at around $x / B=-0.4$, probably due to the high acceleration of the flow induced by the barriers as illustrated for this configuration in the velocity contour plot. In Option 3, there are indications of a small region of separated flow, up to approximately $x / B=-0.2$, suggesting that this option is the least affected by the inclusion of barriers, although the separated region is still much smaller than the basic deck section.

\footnotetext{
${ }^{1} x / B$ represents the non-dimensional horizontal position.
} 


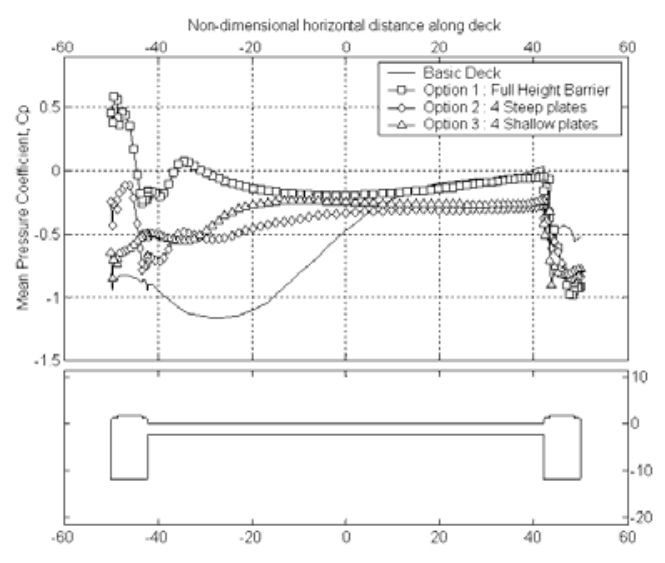

a) Mean pressure coefficient.

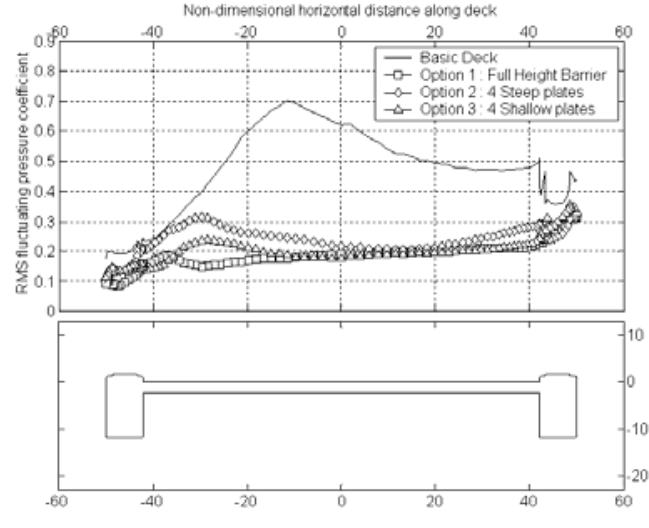

b) RMS fluctuating pressure coefficient Figure 8. Mean and fluctuating pressure coefficients on upper deck surface - Angle of incidence $=0^{\circ}$.

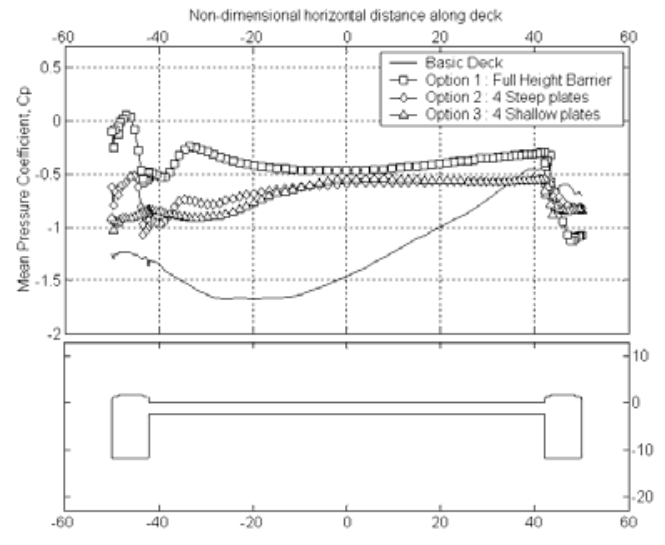

a) Mean pressure coefficient.

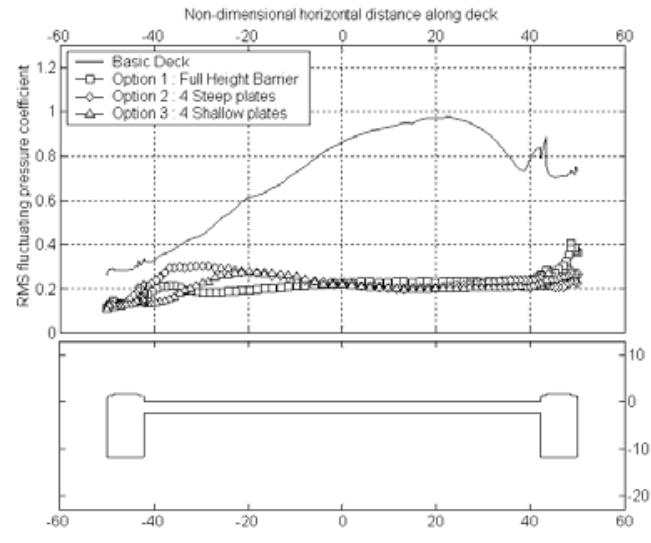

b) RMS fluctuating pressure coefficient.

Figure 9. Mean and fluctuating pressure coefficients on upper deck surface - Angle of incidence $=+5^{\circ}$.

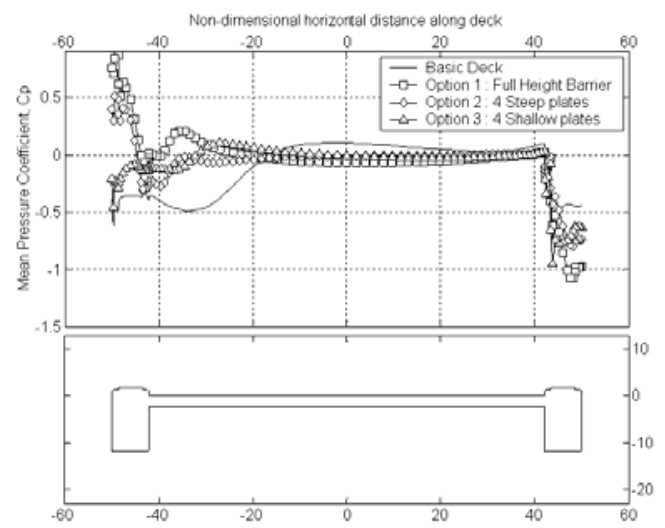

a) Mean pressure coefficient.

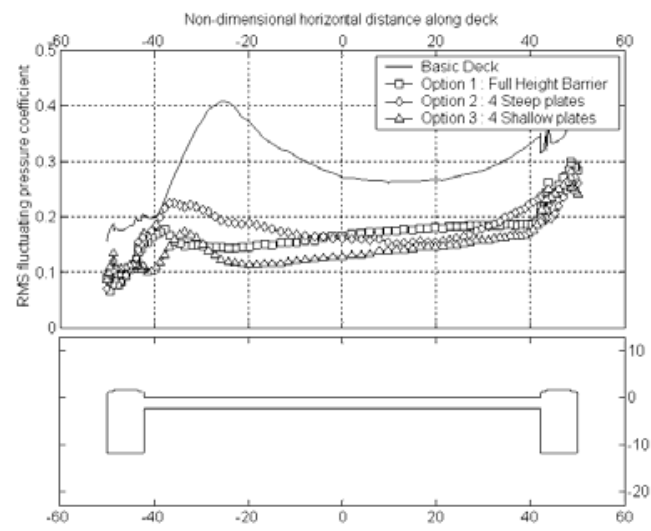

b) RMS fluctuating pressure coefficient.

Figure 10. Mean and fluctuating pressure coefficients on upper deck surface - Angle of incidence $=-5^{\circ}$. 
At $+5^{\circ}$ incidence (Fig. 9), the region of separated flow on the upper surface of the basic deck section is larger than at $0^{\circ}$, extending over the whole of the surface, with large suction pressures and no region of constant pressure towards the rear part of the surface. Also, the fluctuating pressures are generally much higher, especially over the latter half of the deck. In contrast, all three cases with barriers show little difference to the results from the $0^{\circ}$ case. There is a slight shift towards higher suction pressures, probably due to a greater acceleration of the flow around the upper surface of the deck at negative incidence. However, the general shape of the pressure distribution is largely unchanged, with still no indication that large regions of separated flow are present. This is especially the case in Options 1 and 2, although for design Option 3, there is some evidence that the small separation region mentioned earlier has increased slightly in size, now extending up to approximately $x / B=-0.05$ (45\% deck width). The RMS pressure fluctuations are relatively unchanged from the $0^{\circ}$ case, both in terms of the magnitude and the shape of the distribution.

At $-5^{\circ}$ incidence (Fig. 10), the basic deck section shows a smaller separation region, with the flow reattaching at around $x / B=-0.1$ (40\% deck width), also characterised by generally lower RMS pressure fluctuations, associated with wind predominantly along the deck surface suppressing the separation and thus reducing flow unsteadiness. Again, the cases with barriers illustrate that the pressure coefficients are relatively unchanged compared to the $0^{\circ}$ case, with similar distributions indicating almost no region of separated flow. Options 1 and 2 show very little change in the distribution of mean and fluctuating pressure compared to $0^{\circ}$ incidence, indicating that the barriers have the strongest effect in these cases. Option 3 shows that the small separation zone has reduced at $-5^{\circ}$ incidence, with the flow now reattaching at approximately $x / B=-0.3$ to -0.25 (20-25\% deck width), again suggesting that the effect of the barriers is lessened in this case.

\subsection{Proper Orthogonal Decomposition of Unsteady Pressure Field.}

Proper orthogonal decomposition (POD) has been used to analyse the unsteady pressure field on the upper surface of the deck. POD analysis allows the underlying systematic structures within the flow and their relative importance on the overall aerodynamic characteristics to be investigated. Hence, the technique should prove useful in identifying if the "flat plate" barriers are indeed having such a strong effect on the flow over the upper deck surface and the overall aerodynamic forces.

The POD analysis has been applied only to the fluctuating component of the pressure, $\tilde{p}(x, y, t)$ (Eq. 13). As illustrated by Tamura et al. (1999), if the mean value components are included in the analysis, the eigenvectors are distorted by the mean value. Hence, in this analysis, the mean pressures are subtracted from the pressure data.

$$
\tilde{p}(x, y, t)=p(x, y, t)-\bar{p}(x, y) .
$$

For each deck configuration, the first two modes are presented at angles of incidence of $0^{\circ},-5^{\circ}$, and $+5^{\circ}$ in Fig. 11-14. 


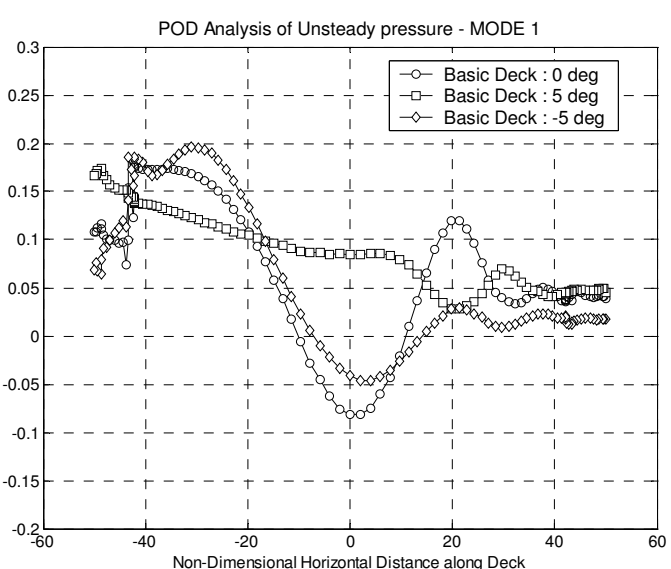

a) POD mode 1 .

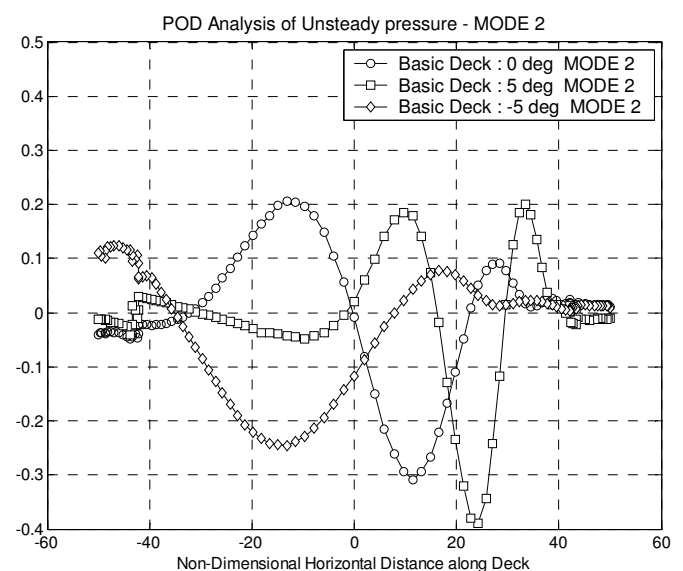

b) POD mode 2 .

Figure 11. First two POD modes of unsteady pressure field on upper surface - Basic Deck.

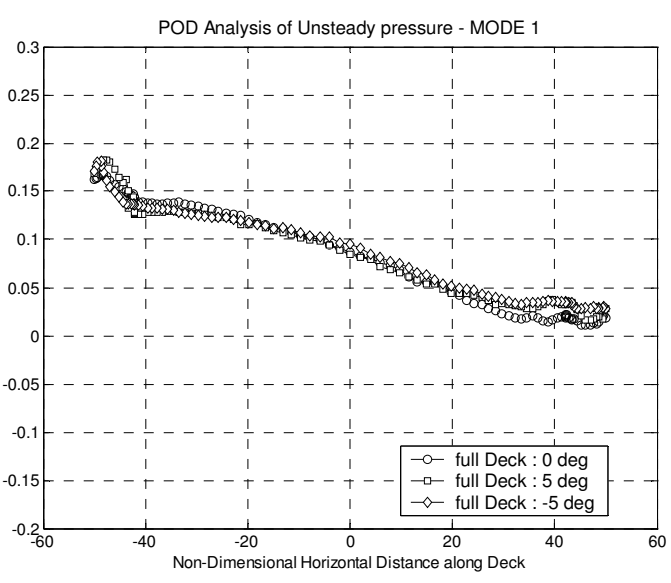

a) POD mode 1 .

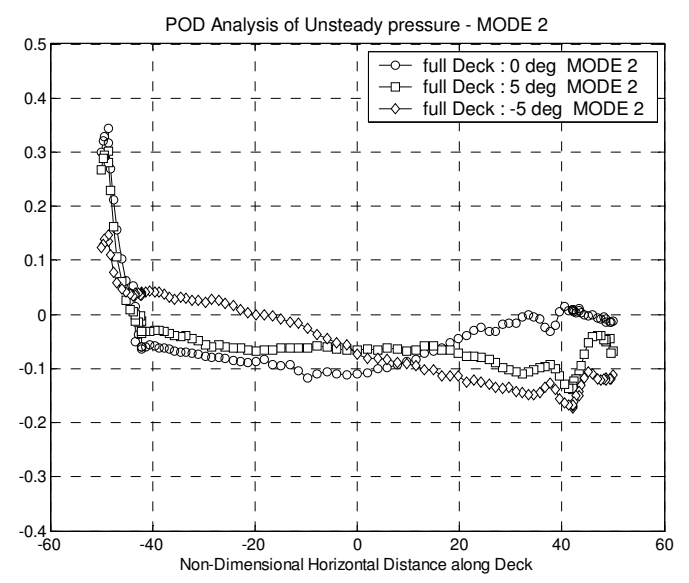

b) POD mode 2 .

Figure 12. First two POD modes of unsteady pressure field on upper surface - Full Barrier, Option 1.

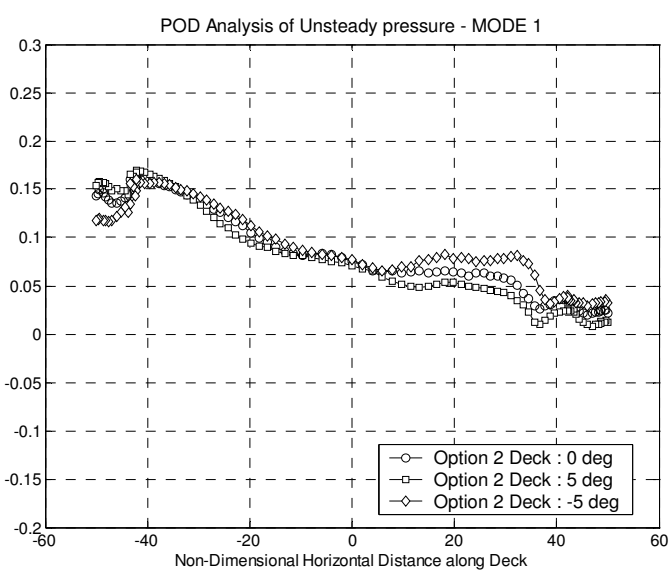

a) POD mode 1 .

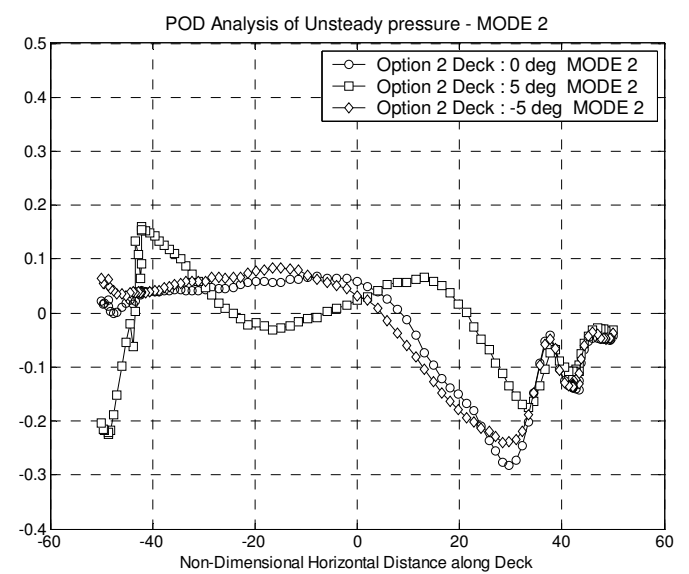

b) POD mode 2 .

Figure 13. First two POD modes of unsteady pressure field on upper surface - Deck Option 2. 


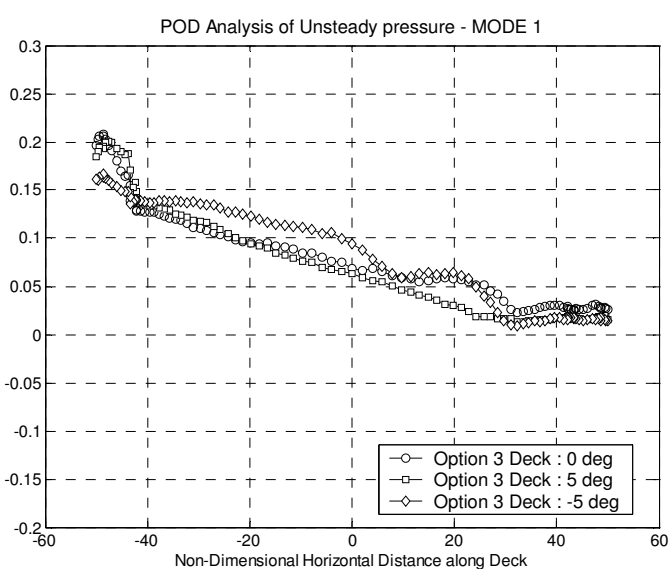

a) POD mode 1 .

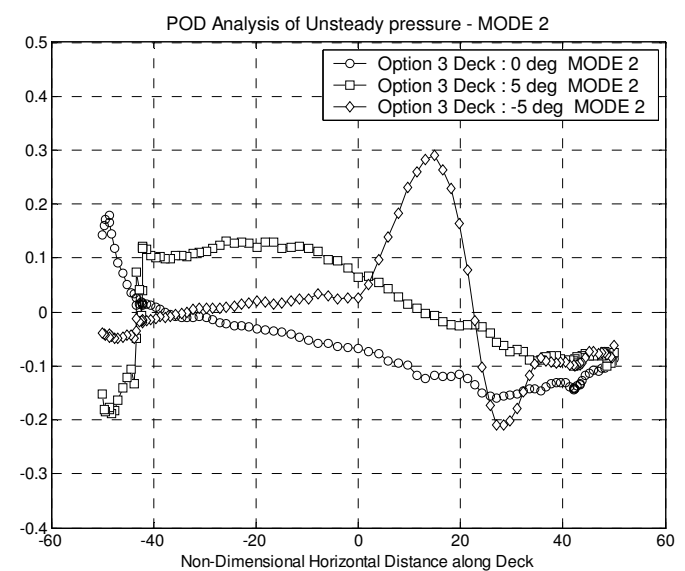

b) POD mode 2.

Figure 14. First two POD modes of unsteady pressure field on upper surface - Deck Option 3.

Table 4. Proportion of principal component.

\begin{tabular}{lcccc}
\hline & $\begin{array}{c}\text { Proportion of } 1^{\text {st }} \\
\text { principal } \\
\text { coordinate }(\%)\end{array}$ & $\begin{array}{c}\text { Proportion of } 2^{\text {nd }} \\
\text { principal } \\
\text { coordinate }(\%)\end{array}$ & $\begin{array}{c}\text { Proportion of } 3^{\text {rd }} \\
\text { principal } \\
\text { coordinate }(\%)\end{array}$ & Eigenvalue sum \\
\hline Basic : $0^{\circ}$ & 32.6 & 21.0 & 15.5 & 19.38 \\
Basic : $+5^{\circ}$ & 47.4 & 10.1 & 9.3 & 8.30 \\
Basic $:-5^{\circ}$ & 37.7 & 26.2 & 12.9 & 46.36 \\
Option $1: 0^{\circ}$ & 48.4 & 8.3 & 7.3 & 3.73 \\
Option $1:+5^{\circ}$ & 46.8 & 9.7 & 8.1 & 3.26 \\
Option $1:-5^{\circ}$ & 50.4 & 9.2 & 7.2 & 4.96 \\
Option $2: 0^{\circ}$ & 39.8 & 12.6 & 8.6 & 5.72 \\
Option $2:+5^{\circ}$ & 35.4 & 11.5 & 10.1 & 3.51 \\
Option $2:-5^{\circ}$ & 36.1 & 16.0 & 11.2 & 5.18 \\
Option 3: $0^{\circ}$ & 52.1 & 8.5 & 5.3 & 4.39 \\
Option 3: $+5^{\circ}$ & 51.8 & 5.7 & 4.7 & 2.26 \\
Option 3: $-5^{\circ}$ & 41.9 & 9.1 & 8.1 & 4.15
\end{tabular}

The POD analysis is a method of finding the most efficient coordinate axis to maximise the energy in terms of the mean square. The physical interpretation of the POD modes is that the coordinate axis ( $1^{\text {st }}$ mode $)$ is determined to maximise the mean square. The $2^{\text {nd }}$ mode is a coordinate axis orthogonal to the $1^{\text {st }}$ mode that maximises the mean square in that direction, likewise for higher modes. As the axis is being determined to maximise the energy of the mean square, vertical axes in Figs. 11-14 provide a measure of energy within the fluctuating flow features. However, as the POD modes essentially represent a direction in state space, the units are somewhat arbitrary. 
The ratio of the $m$ th eigenvalue to the sum of eigenvalues, which gives a measure of the significance of each POD mode, is indicated in Table 4. Also indicated is the sum of eigenvalues for each case. It is clear from these results that the deck options with barriers are dominated by the first mode, and also the sum of eigenvectors are much lower than those for the basic deck section. This could be interpreted as an indication that the flow fields in the cases with barriers show much less unsteadiness, and are dominated more by a particular feature of the flow.

From investigation of the first two POD modes for the basic deck section, the correspondence of these modes with the flow structure over the upper deck surface can be seen. For $0^{\circ}$ incidence, the flow separates at the LE and reattaches at around $x / B=$ 0.1 , as discussed in previous sections, with a peak RMS pressure at about $x / B=-0.1$. Most of the energy for mode 1 is contained within the first $70-75 \%$ of the deck (up to $x / B=0.2$ to 0.25 ), corresponding with the region of separated flow. Also, mode 2 has a positive peak at around $x / B=-0.1$, closely corresponding with the peak RMS pressure fluctuation due to the separation region, and similarly, a negative peak at about $x / B=$ 0.1 corresponding with the reattachment point. The proportion of the first two modes to the sum of eigenvalues are $32.6 \%$ and $21.0 \%$ respectively, indicating that the unsteady separation and reattachment is the major effect on the overall flow field for this case.

For $-5^{\circ}$ incidence, the separation region reattaches earlier, at around $x / B=-0.1$. Most of the energy for mode 1 is again towards the first part of the deck, with only a small amount of energy in the last half of the deck. Mode 2 has a (negative) peak at just less than $x / B=-0.1$, which is consistent with the location of the reattachment. Similar to modes 1 , there is much less variation in the latter half of the deck compared with the $0^{\circ}$ case, due to the much smaller region of separated flow. Although the region of separated flow is smaller, the overall structure of the flow field is similar to the $0^{\circ}$ case, with similar proportions for the first two modes, $37.7 \%$ and $26.2 \%$ respectively, in relation to the sum of eigenvalues.

For the $+5^{\circ}$ case, the flow is separated for almost the whole deck width, which can be seen in mode 1 , with little variation and also most of the energy contained within the first $60 \%$ of the deck (up to $x / B=0.1$ ). Mode 2 also has little variation in the first half of the deck, with most of the energy towards the rear of the deck, corresponding with the peak RMS pressure fluctuation at around $x / B=0.2$ to 0.25 , and also the reattachment point at around $x / B=0.35$ to 0.4 . The dominant feature of the flow field at $+5^{\circ}$ on the upper surface, is the large separation, and this is reflected in the proportions of the first two modes to the overall sum being different to the $0^{\circ}$ and $-5^{\circ}$ cases, with values of $47.4 \%$ and $10.1 \%$ for modes 1 and 2 respectively.

To confirm these interpretations of the POD modes for the basic deck, a frequency analysis of the first two principal coordinates, $a_{n}(t)$, has been performed. Also, frequency analyses were performed on the time history of the lift coefficient of the deck, and also on the unsteady pressure data from the upper deck surface at intervals of $10 \%$ deck width, between $10 \%$ and $90 \%(x / B=-0.4$ to 0.4$)$. 
At $0^{\circ}$ incidence, the lift time history was found to have two dominant peaks at nondimensional frequencies 0.425 and 0.848 , with the second of these being slightly more dominant. The higher frequency peak corresponds to the vortex shedding from the rear of the deck, and the lower frequency peak is due to the effects of the unsteady shear layers separating from the leading edge of the deck. Frequency analysis of the first principal coordinate also exhibits the same characteristics, with the peak at 0.425 being the most dominant. However, in the second principal coordinate, the higher frequency component is not significant, and a dominant peak at a frequency of 0.49 is now present. Frequency analysis of the unsteady pressures from $10 \%$ to $90 \%$ deck width, demonstrate that the peak at a frequency of 0.49 only appears in the results between $40 \%-70 \%$ deck width $(x / B=-0.1$ to 0.3$)$, and is the dominant frequency between 50 $60 \%(x / B=0$ to 0.1$)$ (Fig. 15). This corresponds closely with the reattachment point and confirms the interpretation of the POD modes.

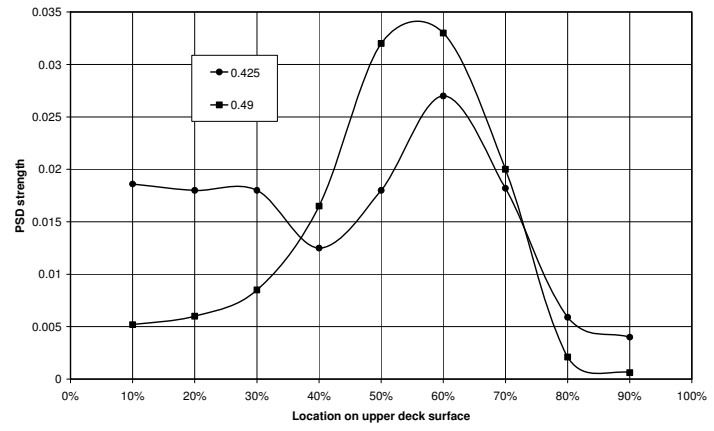

a) Basic deck : $0^{\circ}$.

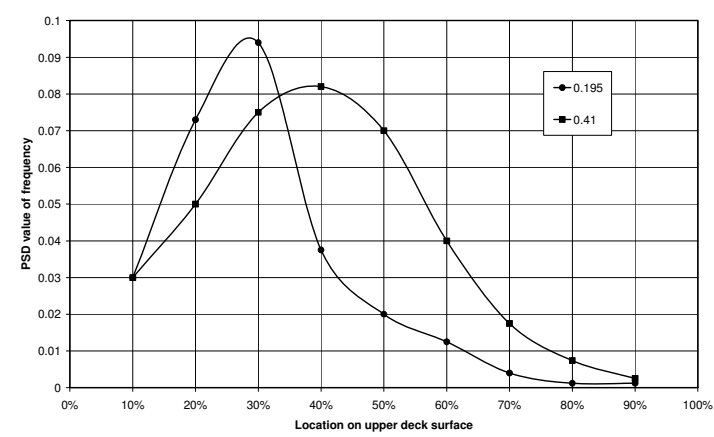

c) Basic deck : $-5^{\circ}$.

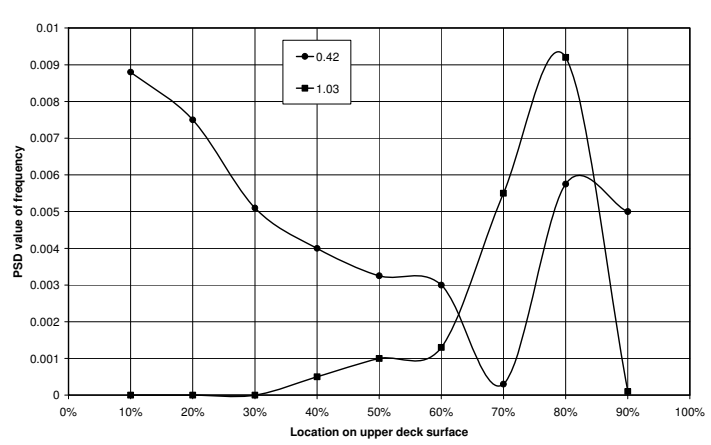

b) Basic deck : $+5^{\circ}$.

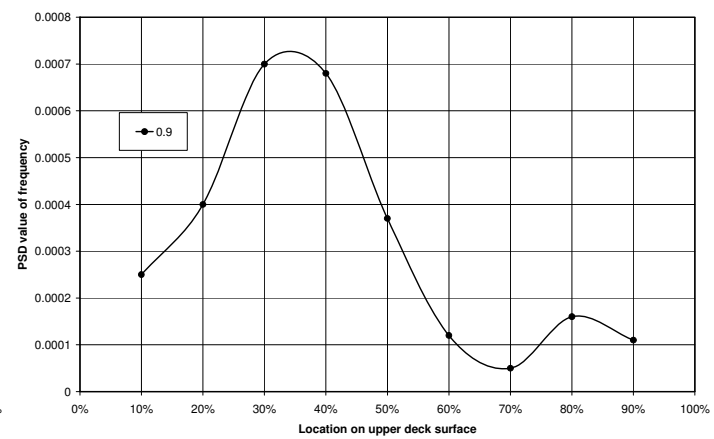

d) Option $1: 0^{\circ}$.

Figure 15. Frequency analysis of unsteady pressures on upper deck surface : Power spectral density of dominant frequencies.

A similar analysis for the $+5^{\circ}$ and $-5^{\circ}$ cases also confirm the POD interpretation. At $+5^{\circ}$, the lift is dominated by a single component at a frequency of 0.42 , due to the unsteady wake separating from the leading edge of the deck. The first principal coordinate is also dominated by this frequency component, whereas the second is dominated by a frequency of 1.03 . Analysis of the pressures confirm that this higher frequency component only arises in the last $30 \%$ of the deck $(x / B=0.2$ onwards), and is due to the intermittent reattachment of the flow at the rear part of the deck, consistent 
with the interpretation of the POD modes. In the first part of the deck, the component at a frequency of 0.42 is dominant (Fig. 15).

For the $-5^{\circ}$ case, the orientation of the deck mean that the separation at the leading edge is small, and the flow is largely attached over the upper deck surface, and so unsteadiness in the lift coefficient is more likely to arise from flow features on the underside of the deck. Hence, correspondence between dominant frequencies in the lift coefficient and the principal coordinates are harder to determine. However, the first principal coordinate has a dominant component at a frequency of 0.195 , which is similarly observed from the pressure frequency analysis at around 20-30\% deck width $(x / B=-0.3$ to -0.2$)$, corresponding to the separation region. The second principal coordinate has a dominant component at a frequency of 0.41 , which is observed from the pressure frequency analysis at around $40 \%$ deck width $(x / B=-0.1)$, corresponding to the reattachment point (Fig. 15). These results are consistent with the POD analysis discussed earlier.

In all cases with the barriers, particularly option 1 with full height barriers, mode 1 shows very little variation for different angles of incidence. Also, there is less energy associated with these modes compared to the basic deck, giving an indication that the fluctuations in the flow field are much lower in this case. Comparing this result with those for the basic deck discussed above, the lack of variation of the POD modes with angle of incidence demonstrates clearly the strong effect the barriers are having on the overall flow field. In all cases with barriers, the first mode provides by far the dominant contribution to the overall sum of eigenvalues, and has similar values for all angles of incidence, unlike the basic deck section (Table 4). For each barrier option, the contribution of mode 1 is of the order $40 \%$ to $50 \%$, whereas for mode 2 the proportion is much lower at around 8-15\%. A slightly larger variation is noted for option 3 , which is possibly an indication that this configuration is less affected by the barriers, a feature also noted from separate investigation of the mean pressure distributions.

As discussed earlier, the barrier effects virtually remove the separation region, hence there is also very little variation in RMS pressure fluctuations. This can also be noted in POD mode 2 and 3 for the cases with barriers, with much less variation and energy when compared to the basic deck. Deck options 2 and 3 show a little more variation for mode 2, particularly towards the rear of the deck. This arises as the upstream barriers for options 2 and 3, with their reduced height, tend to have the strongest effect on the fore region of the deck. However, as the angle of incidence varies, the flow over the top of the barriers can still interact with the downstream portion of the deck. The largest variation is for option 3 at $+5^{\circ}$, where the positive incidence and reduced barrier angle allow a separation region to develop, and the peak in mode 2 at about $x / B=0.15$ corresponds to the reattachment point of this separation (Fig. 16).

Frequency analysis of the lift time history, unsteady pressures and principal coordinates for deck Option 1 at $0^{\circ}$ are much more difficult to interpret as many of the unsteady flow features on the upper surface of the deck have been suppressed by the barriers. The lift coefficient has a reasonably dominant component at a frequency of 
0.48. It is likely that this is influenced by flow features on the underside of the deck, as a peak at this frequency does not appear in any of the frequency analyses of the upper surface pressures. The frequency analysis of the surface pressures are quite noisy, with generally no dominant frequency component, except for a distinct peak at a frequency of 0.9 , at $x / B=-0.3$ to -0.1 (Fig. 15). This is also the dominant frequency in the frequency analysis of the first principal coordinate, with the second and third principal coordinates not displaying any significant features. The flow is being directed downwards by the barriers at the leading edge and, from Figs. 5-6, is impinging on the surface at this location. Hence, the interpretation of the POD modes is confirmed, that their uniformity with angle of incidence and low energy is an indication that the flow over the upper surface is dominated by the barriers. Similar effects can be determined for the other two barrier configurations.

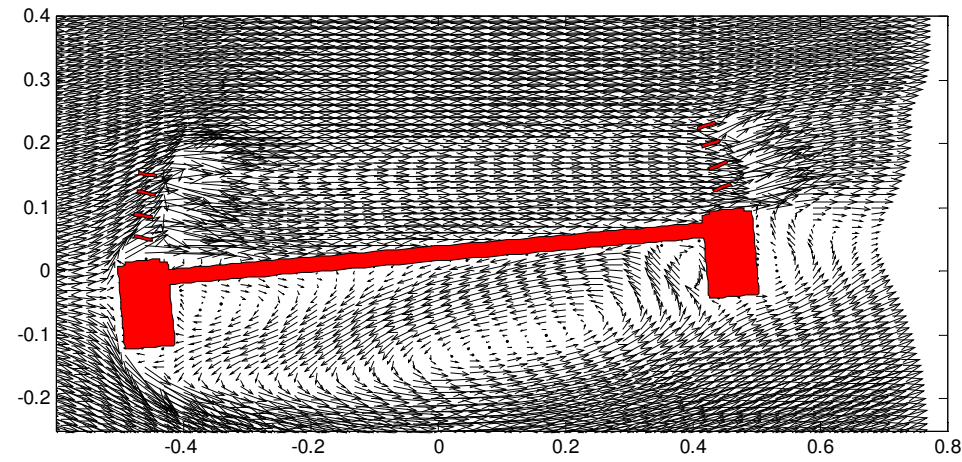

a) $-5^{\circ}$ incidence.

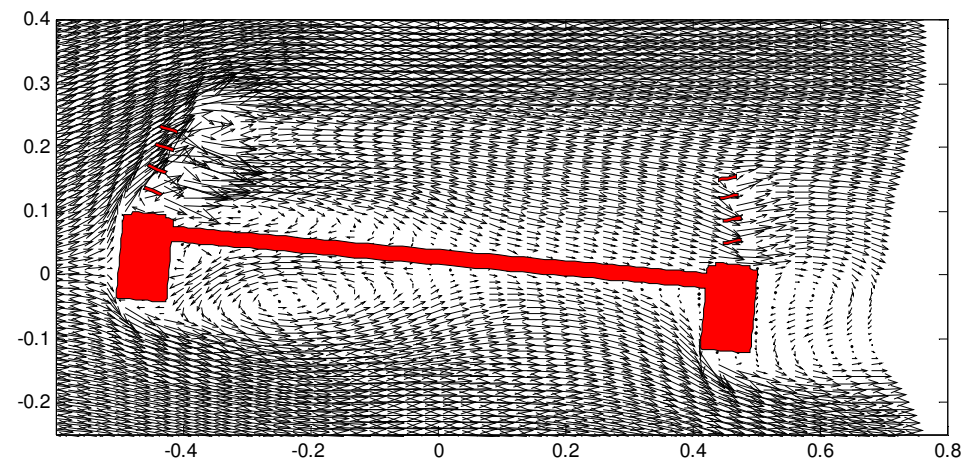

b) $+5^{\circ}$ incidence.

Figure 16. Deck option3 at non zero angles of incidence - Mean velocity vectors.

\section{DYNAMIC ANALYSIS}

In the dynamic analysis, determination of the flutter instability was investigated through a forced oscillation technique, using the traditional flutter derivatives (Simiu and Scanlan, 1996), with the lift and moment defined as : 


$$
\begin{aligned}
& L=\frac{1}{2} \rho U_{\infty}^{2}(2 B)\left[k H_{1}^{*}(k) \frac{\dot{h}}{U_{\infty}}+k H_{2}^{*}(k) \frac{B \dot{\alpha}}{U_{\infty}}+k^{2} H_{3}^{*}(k) \alpha+k^{2} H_{4}^{*}(k) \frac{h}{B}\right] \\
& M=\frac{1}{2} \rho U_{\infty}^{2}\left(2 B^{2}\right)\left[k A_{1}^{*}(k) \frac{\dot{h}}{U_{\infty}}+k A_{2}^{*}(k) \frac{B \dot{\alpha}}{U_{\infty}}+k^{2} A_{3}^{*}(k) \alpha+k^{2} A_{4}^{*}(k) \frac{h}{B}\right],
\end{aligned}
$$

where the reduced frequency is defined as $k=\frac{B \omega}{U}$.

The bridge deck motion, in either the vertical or torsional DOF, was modelled using a series of forced sinusoidal oscillations over a range of reduced frequencies. From this series of calculations, the flutter derivatives have been ascertained from spectral analyses of the time histories of the predicted unsteady lift and moment coefficients. During this analysis, the amplitude of the sinusoidal motion was $5 \%$ of the bridge deck width, $B$, in the vertical degree of freedom, and $4^{\circ}$ in the torsional degree of freedom.

In this investigation, it was found that the effect of the barriers on the aeroelastic stability of the bridge was to make the structure more susceptible to single degree of freedom flutter instability in the torsional direction, similar to that experienced by the Tacoma Narrows bridge (Billah and Scanlan, 1991). Hence, only 1DOF torsional flutter is considered in this analysis, rather than classical 2DOF flutter. To assess the critical flutter speed of the bridge for 1DOF torsional flutter, a critical value of $A_{2}{ }^{*}$, related to the "aerodynamic damping", can be ascertained from the equation of motion in the torsional degree of freedom.

$$
A_{2}^{*} \text { crit }=\frac{2 I_{\alpha} \zeta_{\alpha}}{\rho B^{4}}
$$

where $I_{\alpha}$ is the mass moment of inertia and $\zeta_{\alpha}$ is the critical damping ratio, and it is assumed that the critical flutter frequency is equal to the fundamental torsional frequency, $\omega_{\alpha}$.

The critical flutter speed for 1DOF torsional flutter is the wind speed at which the "system damping" (combined structural and aerodynamic damping) is zero, and beyond this speed, that is at values higher than the critical $A_{2}{ }^{*}$, a "negative damping" criterion arises. Using the structural properties given in Table 2, a critical value of $A_{2}{ }^{*}$ crit $=0.0459$ for the onset of 1DOF torsional flutter can be derived.

\subsection{Flutter analysis of proposed design options}

As stated earlier, the effect of the barriers on the aeroelastic stability of the bridge was to make the structure more susceptible to single degree of freedom flutter instability in the torsional direction. Hence, for each deck option only the results for the flutter derivative $A_{2}{ }^{*}$ are presented in Fig. 17, with the critical reduced velocities and wind speeds for each deck option shown in Table 5. The wind speeds are all normalised with respect to the design wind speed for the proposed bridge. It should be noted that the lowest reduced velocity used in the DIVEX analysis was 3.0, and critical speeds lower than this were determined by using a spline curve fit to the data. 


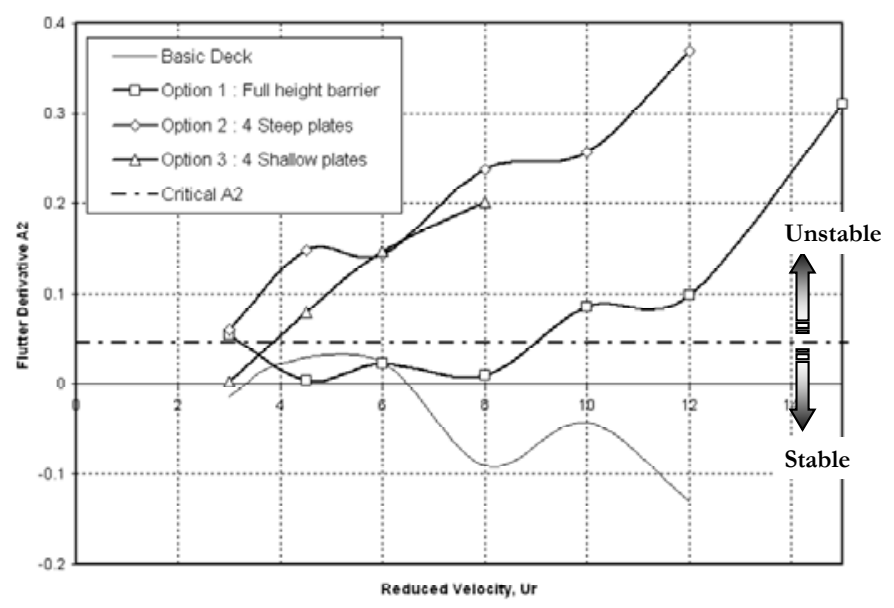

Figure 17. $A_{2}{ }^{*}$ "Torsional Aerodynamic Damping” Flutter Derivatives for each Deck Option Comparison with Critical Value.

Table 5. Critical speeds of single DOF flutter instability for Each Bridge Option.

\begin{tabular}{lcc}
\hline Configuration & $\begin{array}{c}\text { Reduced velocity for } \\
A_{2, \text { crit }}^{*}\end{array}$ & $\begin{array}{c}\text { Critical flutter speed } \\
\text { (normalised wrt design } \\
\text { speed) }\end{array}$ \\
\hline Option $1:$ Full height flat plates. & 1.25 & 0.381 \\
Option $2: 4$ steep flat plates. & 2.74 & 0.838 \\
Option $3: 4$ shallow flat plates. & 3.87 & 1.186 \\
$\begin{array}{l}\text { Option } 3: \text { Using structural properties of } \\
\text { Deck A. }\end{array}$ & 4.088 & 2.733 \\
$\begin{array}{l}\text { Deck A } \\
\text { Deck A : Using structural properties of } \\
\text { proposed bridge. }\end{array}$ & 4.682 & 3.176 \\
\hline
\end{tabular}

For the basic deck section, the $A_{2}{ }^{*}$ derivative did not exceed the critical value, although it is close to becoming unstable at a reduced velocity of approximately 5 , which would correspond to a normalised critical speed 1.53. From further analysis of the remaining flutter derivatives for the basic deck section, a coupled 2 degree of freedom flutter instability was found to occur at a reduced velocity of 9.64, with a nondimensional critical speed of 2.69, well in excess of the design speed. For the basic deck, this critical speed is well in excess of the design speed, indicating that the longer than usual span of this deck does not cause any concern with respect to the aeroelastic stability of the bridge.

In each of the deck options with barriers, $A_{2}{ }^{*}$ is either positive or close to zero, and the critical value is exceeded in the range of reduced velocities considered, indicating a greater susceptibility to single DOF torsional flutter when barriers are included on the 
deck. From the static analysis, the full height barriers used in Option 1, was found to have the strongest effect on the flow, and this is confirmed in the flutter analysis, with this configuration being found to be the most susceptible to a 1DOF torsional instability, initiated at a critical speed as low as $38 \%$ of the design wind speed. However, the deck does become stable once more at a reduced velocity just over 3.0, corresponding to a normalised critical speed of approximately 1.0.

Option 2 with reduced height barriers still proved to be very susceptible to instability, with a 1DOF torsional flutter instability found at a critical speed of $84 \%$ of the design speed. The susceptibility of the bridge to flutter is still strongly dominated by the effect of the barriers on the flow, although reducing the number of flat plates has improved the stability of the bridge, with a significant increase in the critical speed. However, the flutter instability is still well below the design speed.

Option 3 kept the reduced number of flat plates, but significantly reduced their angle to the deck, to further moderate the turning effect of the barriers at the edges of the deck. From the static analysis, the barriers in this configuration were found to still have a strong effect on the flow, but not to the same extent as the design options 1 and 2 . This modification successfully increased the flutter speed to a value above the design speed. Although the critical speed is significantly lower than for the basic deck, the 1DOF torsional flutter instability now occurs at a speed $19 \%$ higher than the design wind speed.

\subsection{Validation of flutter analysis.}

Extensive validation of DIVEX for determining aerodynamic characteristics and flutter derivatives for bluff bodies has been previously undertaken, extensive details of which are given elsewhere (Taylor and Vezza, 2001, 2002a; Taylor et al. 2002b). For example, flutter derivatives on streamline and bluff deck sections have been successfully predicted and critical flutter speeds have been accurately determined to within a few $\mathrm{m} / \mathrm{s}$. Also, DIVEX has been used previously in design studies for bridge deck flutter, providing accurate results for both static and dynamic aerodynamic characteristics, sometimes in advance of experimental details being available. These results give confidence in the numerical method, to give a reliable prediction on a new geometry where no previous information or experimental data is available.

The investigation of this new bridge, by definition, is an extension to previous work and hence there is limitation on the particular validation of this configuration. However, structural properties and wind tunnel results of a previous bridge design, incorporating a similar barrier configuration, were used to investigate how DIVEX performs on this type of configuration. Due to commercial confidentiality this alternative bridge is simply labelled "Deck A". Importantly, this bridge had not experienced the sensitivity to the barriers and had not demonstrated a strong flutter instability either in wind tunnel tests or in service.

A brief numerical investigation into Deck A was performed using DIVEX, in an attempt to understand these results, and also to assess why Deck A appears to be so 
much more stable, with the results summarised in Table 5 and Fig. 18. From the DIVEX predictions of the flutter derivatives for Deck A, a non-dimensional critical flutter speed of 3.176 was predicted. Wind tunnel tests indicated that Deck A would be stable up to a non-dimensional speed of around 1.7, with no critical speed being determined. The DIVEX results are therefore qualitatively in agreement with the wind tunnel results, that Deck A appears to be a much more stable structure.

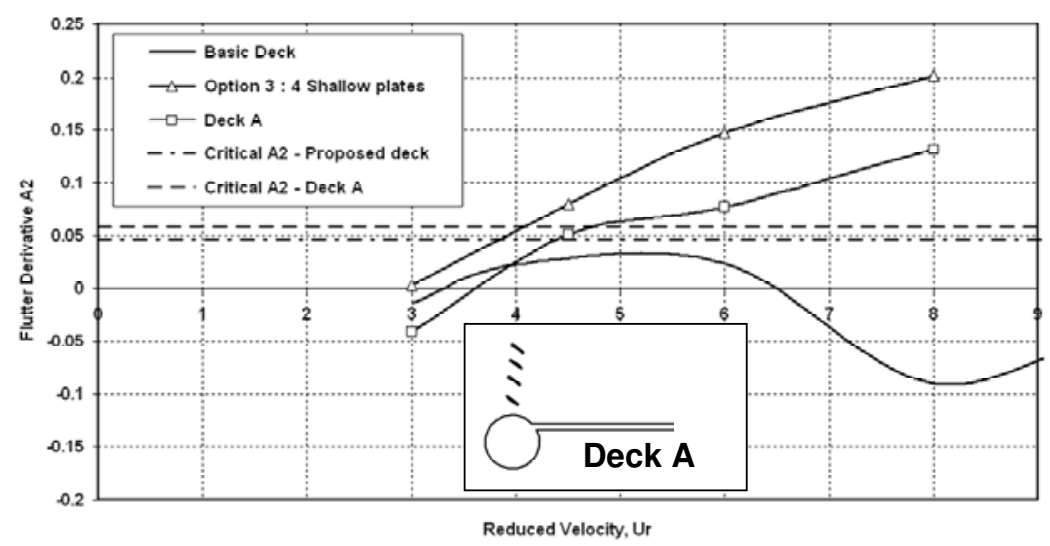

Figure 18. Comparison of A2* Flutter Derivative and critical values for proposed deck section and alternative Deck A.

It was ascertained that two main factors contribute to the greater stability of Deck A. Firstly, Deck A has very different structural characteristics to the proposed new bridge, with a wider and heavier deck section, significantly stiffer, with higher moment of inertia and fundamental frequencies ( $f_{\alpha}$ is more than doubled). These differences, in particular the higher frequency, reduce the susceptibility of the structure to flutter instabilities, increasing the critical value of $A_{2}{ }^{*}$ at which there is zero system damping. The effect of the different structural properties is confirmed when the flutter derivatives predicted by DIVEX for Deck A are used along with the structural properties for the new proposed bridge. The non-dimensional critical speed at which Deck A would exhibit 1DOF torsional flutter is more than halved, to a value of 1.338 (Table 5). Similarly, if the structural properties of Deck A are applied to the new proposed bridge, the flutter speed is more than doubled, from 1.186 to 2.773 for deck Option 3. Clearly, the different structural properties, particularly the increased deck width and torsional frequency, have a strong effect on the flutter stability, however, this critical speed, from a barrier configuration with steep angled plates, is still higher than the best flutter speed achieved on the new bridge.

The second factor contributing to the greater stability of the alternative deck the different arrangement of the leading edge. For the new bridge, the deck is essentially a flat plate between two rectangular sections, whereas Deck A is a flat plate between two circular sections (Fig. 18). The different arrangements at the leading edge have a significant effect on the flow around the basic deck sections, and hence on the flow approaching the barriers. The rectangular section for the new deck deflects the flow upwards, causing a large separation region to develop on the upper deck surface (Fig. 
19). For Deck A, the rounded leading edge means that the shear layer over the upper surface separates at a significantly lower angle, thus greatly reducing the size of the separated flow region. When the barriers are included, the flow over the alternative deck has a much larger horizontal component due to the circular leading edge, and so the barriers do not have such a dominant effect on the flow over the upper surface of the deck. These differences have the effect of improving the aerodynamic damping for the deck, with the $A_{2}{ }^{*}$ derivative becoming positive at higher reduced velocities for Deck A. Hence Deck A is not as susceptible to the 1DOF flutter instability.

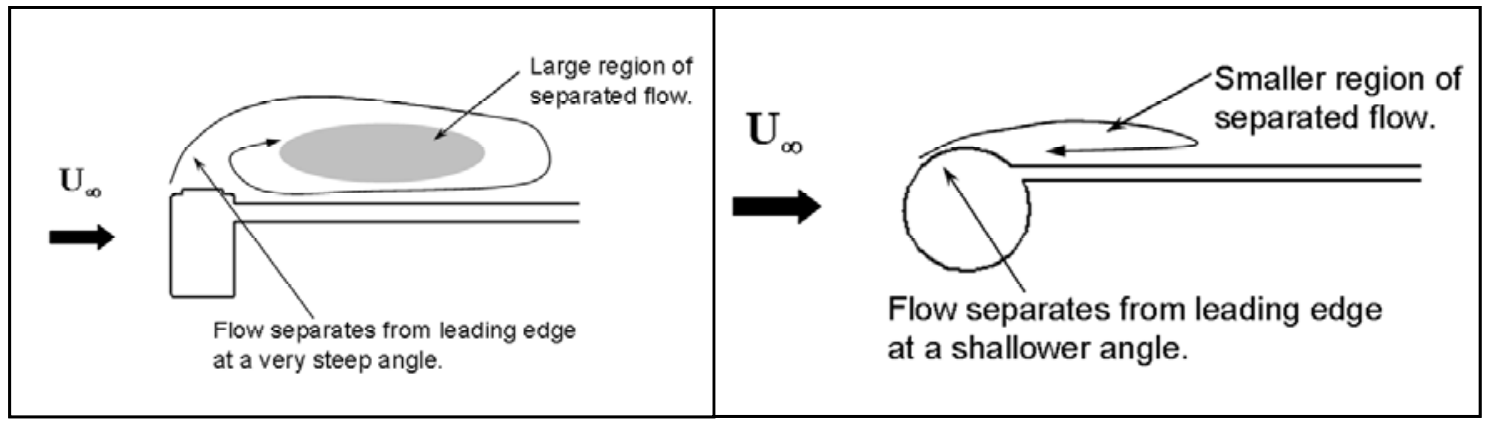

Figure 19. Schematic diagram of flow around leading edge of new bridge deck and Deck A.

In this discussion, we have not taken account of any Reynolds number dependency of the results for Deck A due to the rounded leading edge. However, at wind speeds above the design speed, the Reynolds number based on the across wind dimension of the deck is $O\left(10^{6}\right)$ in the post-critical region for circular cylinders. For this reason, the separation point is likely to be close to the top of the rounded leading edge, and the shear layer is less likely to be deflected up towards the barriers as highlighted in the earlier qualitative discussion.

Although this does not provide a full validation of DIVEX for this configuration, the qualitative agreement of the results with the experimental information from the previous deck provides some additional measure of confidence in the numerical approach.

\section{CONCLUSIONS}

A numerical study into the aerodynamic characteristics and aeroelastic stability of a proposed footbridge has been undertaken, with the main conclusions being :

- The vortex method, DIVEX, has been demonstrated as a useful and effective design tool for assessing candidate bridge deck configurations, allowing various design options to be considered.

- The inclusion of angled flat plates at the edges of the deck section has a strong effect on the aerodynamic characteristics of the deck.

- Flow visualisation demonstrates the flat plates acting strongly as flow guide vanes, directing the flow downwards at the leading edge and upwards at the trailing edge.

- The flat plates have a strong effect on the static aerodynamic loads of the sections - lower lift, higher drag and moment at $0^{\circ}$, as well as an order of magnitude reduction in moment slope. 
- Proper orthogonal decomposition has been used to illustrate the strong effect of the pedestrian barriers on the flow field over the upper deck surface of the bridge. Results are in accordance with the assessments based on the velocity field visualisation and the RMS pressures.

- The basic deck section, with no flat plates, experiences a two degree of freedom flutter instability, with a critical flutter speed well in excess of the design speed.

- Including the pedestrian barriers significantly increases the susceptibility of the footbridge to a flutter instability.

- Critical flutter speed of the deck sections are strongly affected by the configuration and angle of flat plates. Deck options 1 and 2, both with steeply angled flat plates have critical speeds, or are unstable below the design wind speed.

- Deck option 3, with a small number of shallow angled flat plates is stable with respect to flutter up to a wind speed approximately $19 \%$ greater than the design wind speed. In conjunction with a built-in safety margin, this was considered to be sufficiently far from the design speed to be a viable design option.

- An alternative deck section based on a previous design was considered, and was found to have a critical flutter speed $34 \%$ greater than the design wind speed. 


\section{REFERENCES}

Akbari, M.H., Price, S.J., 2003. Simulation of dynamic stall for a NACA 0012 airfoil using a vortex method. Journal of Fluids and Structures, 17, 855-874.

Akbari, M.H., Price, S.J., 2005. Numerical investigation of flow patterns for staggered cylinder pairs in cross-flow. Journal of Fluids and Structures, 20, 533-554.

Amandolèse, X., Crémona, C., 2005. Analysing fluid loadings on moving bluff bodies using proper orthogonal decomposition. Journal of Fluids and Structures, 20, 577-587. Billah, K.Y., Scanlan, R.H., 1991. Resonance, Tacoma Narrows Bridge Failure, and Undergraduate Physics Textbooks. American Journal of Physics, 59(2), 118-124 Chen, L., Letchford, C.W., 2005. Proper orthogonal decomposition of two vertical profiles of full-scale wind nonstationary downburst wind speeds. Journal of Wind Engineering and Industrial Aerodynamics, 93, 187-216.

Chen, X., Kareem, A., 2005. Proper orthogonal decomposition-based modelling, analysis, and simulation of dynamic wind load effects on structures. Journal of Engineering Mechanics, 131(4), 325-339.

Larsen, A., Walther, J.H., 1997. Aeroelastic Analysis of Bridge Girder Sections Based on Discrete Vortex Simulations. Journal of Wind Engineering and Industrial Aerodynamics, 67-68, 253-265.

Leonard, A., 1980. Vortex Methods for Flow Simulation. Journal of Computational Physics, 37, 289-335.

Leonard, A., 1985. Computing Three-Dimensional Incompressible Flows with Vortex Elements. Annual Review of Fluid Mechanics, 17, 523-559.

Lin, H., 1997. Prediction of Separated Flows Around Pitching Aerofoils using a Discrete Vortex Method. Ph.D. Thesis 10896, Department of Aerospace Engineering, University of Glasgow, Scotland, UK

MacKenzie, D.K., Morgenthal, G., McRobie, F.A., 2002. Wind shielding of the M4 at Neath. Proceedings of 5th UK Conference on Wind Engineering, Nottingham, U.K., 46 September 2002.

Matsumoto, M., Yagi, T., Tubota, T., Lee, J.-H., Hori, K., Kawashima, Y., 2006. Study on the unsteady pressure field around the oscillatory $\mathrm{B} / \mathrm{D}=4$ rectangular section using proper orthogonal decomposition analysis. Proc. of $4^{\text {th }}$ Int. Symp. on Computational Wind Engineering, 16-19 July 2006, Yokohama, Japan.

Robertson, A., Taylor, I.J., 2006. Numerical analysis of forced oscillations of a circular cylinder using a discrete vortex method. Proceedings of $7^{\text {th }}$ UK Conference on Wind Engineering, 4-6 September 2006, Glasgow, UK, 135-138.

Sarpkaya, T., 1989. Computational Methods with Vortices - The 1988 Freeman Scholar Lecture. Journal of Fluids Engineering, 111, 5-52.

Sweeney, C., Meskell, C., 2003. Fast numerical simulation of vortex shedding in tube arrays using a discrete vortex method. Journal of Fluids and Structures, 18, 501-512. 
Tamura, Y., Suganuma, S., Kikuchi, H., Hibi, K., 1999. Proper orthogonal decomposition of random wind pressure field. Journal of Fluids and Structures, 13, 1069-1095.

Simiu, E., Scanlan, R.H., 1996. Wind Effects on Structures : Fundamentals and Applications to Design. 3rd Edition, John Wiley and Sons.

Taylor, I.J., Vezza, M., 1999a. Prediction of unsteady flow around square and rectangular section cylinders using a discrete vortex method. Journal of Wind Engineering and Industrial Aerodynamics, 82(1-3), 247-269.

Taylor, I.J., Vezza, M., 1999b. Calculation of the Flow Field around a Square Section Cylinder undergoing Forced Transverse Oscillations using a Discrete Vortex Method. Journal of Wind Engineering and Industrial Aerodynamics, 82(1-3), 271-291.

Taylor, I.J., Vezza, M., 2001. Application of a discrete vortex method for the analysis of suspension bridge deck section. Wind and Structures, 4(4), 333-352.

Taylor, I.J., Vezza, M., 2002a. Aeroelastic stability analysis of a bridge deck with added vanes using a discrete vortex method. Wind and Structures, 5(2-4), 277-290. Taylor, I.J., Vezza, M., Withycombe, S.I., 2002b. Commercial computational wind engineering : Contributions of DIVEX to recent design projects. Proceedings of 5th UK Conference on Wind Engineering, Nottingham, U.K., 4-6 September 2002.

Taylor, I.J., Vezza, M., Salisbury, I., 2005. A study into the performance of wind shields on both streamlined and bluff bridge deck sections. Proc. of $4^{\text {th }}$ European and African Conference on Wind Engineering, Prague, Czech Republic, 11-15 July 2005. The Highways Agency, 2001. Design Rules for Aerodynamic Effects on Bridges. BD 49/01, Vol. 1, Sect. 3, Part 3.

Vejrum, T., Queen, D.J., Larose, G.L., Larsen, A., 2000. Further aerodynamic studies of Lions' Gate Bridge - 3 lane renovation. Journal of Wind Engineering and Industrial Aerodynamics, 88(2-3), 325-341. 